\title{
Radiative Effects of Cirrus Clouds
}

\author{
By \\ James R. Fleming \\ Department of Atmospheric Science \\ Colorado State University \\ Fort Collins, Colorado
}

\section{Department of \\ Atmospheric Science}

Paper No. 207 
RADIATIVE EFFECTS OF CIRRUS CLOUDS

by

James R. Fleming

This report was prepared with support from The National Science Foundation

Grant No. GA-36302

Principal Investigator: Stephen K. Cox

Department of Atmospheric Science

Colorado State University

Fort Collins, Colorado

August 1973

Atmospheric Science Paper No. 207 
ABSTRACT

RADIATIVE EFFECTS OF CIRRUS CLOUDS

The divergence of net radiation in a tropical atmosphere with cirrus clouds has been examined in terms of two bulk radiative properties of the cloud: shortwave optical thickness, $\tau^{*}$, and broad-band infrared emissivity, $\varepsilon^{*}$. The shortwave optical thickness of the cirrus cloud is the primary factor controlling the radiative energy budget at the earth's surface while the cloud's broad-band infrared emissivity is the primary factor influencing the heat budget of the atmosphere.

The net radiative energy budget of the tropical atmosphere over an ocean surface in the presence and absence of a cirrus cloud has been. examined. The total net radiative energy at the top of the atmosphere is relatively unchanged from the clear sky value by the presence of a cirrus cloud layer while the surface energy budget shows a significant decrease in the shortwave component. The total net radiative energy loss of the atmosphere when a cirrus layer is present is shown to be $22 \%$ to $78 \%$ of the loss from a cloudless sky. This suppression of divergence generates in 24 hours an amount of energy which is $32 \%$ to $99 \%$ of the estimated net latent heat released to the atmosphere by precipitation processes in a cumutonimbus cloud.

A parameterization technique has been developed which gives radiative temperature change as a function of cloud base temperature and geometric thickness for three layers encompassing the atmosphere: (1) the surface to cloud base layer, (2) the cloud layer itself, (3) the layer from cloud top to the top of the atmosphere. The divergence of net radiation through these three layers is shown to be 
Tinearly dependent on the cirrus cloud's infrared emissivity and shortwave optical thickness. Radiative warming in the cirrus layer itself increases with increasing $\varepsilon^{*}$ and with decreasing cloud temperature.

James R. Fleming

Department of Atmospheric Science Colorado State University

Fort Collins, Colorado 80521 August, 1973 


\section{ACKNOWLEDGEMENTS}

I extend my sincere thanks to $\mathrm{Dr}$. Stephen $\mathrm{K}$. Cox for his guidance during my graduate studies. Dr. Thomas H. Vonder Haar, Dr. David A. Krueger and Dr. Roger M. Gallet have made many helpful suggestions concerning this research. A special thank you is due $\mathrm{Dr}$. Thomas B. Mckee for his advice on theoretical problems. The programming ability and optimistic attitude of M. Charlene Polifka are sincerely appreciated.

This research has been supported by the Atmospheric Sciences Section of the National Science Foundation, Grant GA-36302. 
Page

ABSTRACT

ACKNOWLEDGEMENTS

iv

TABLE OF CONTENTS

LIST OF TABLES

vi

LIST OF FIGURES

vii

LIST OF SYMBOLS

ix

SUBSCRIPTS

$x i$

I. INTRODUCTION

II. PROPERTIES OF CIRRUS CLOUDS 3

A. Observed Physical Properties 3

B. Shortwave Radiative Properties 4

C. Longwave Radiative Properties $\quad 14$

III. THE CLOUD MODEL 18

A. Shortwave Radiative Transfer 18

B. Longwave Radiative Transfer 21

IV. MODEL RESULTS 26

A. Radiative Divergence 26

B. Parameterization of Cirrus Effects on
Radiative Divergence

C. Radiative Energy Budget of the Tropical Atmosphere 42

v. CONCLUSION 56

LITERATURE CITED

APPENDIX A: Cloud layer longwave radiative temperature
change as a function of $\varepsilon^{*}$

APPENDIX B: Albedo of the sea surface for Global Radiation 


\section{LIST OF TABLES}

Table

1 Geometric, physical and radiative parameters used in deriving the radiative effects of cirrus clouds . . . . 17

2 Model cirrus cloud characteristics ......... 26

3 Combinations of $\tau^{*}$ and $\varepsilon^{*}$ which give the maximum radiative temperature change for three layers of the

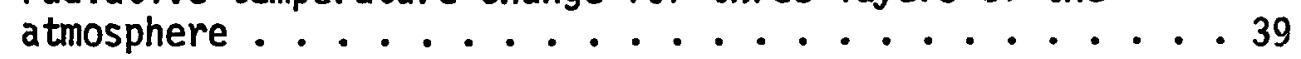

4 Divergence of total radiation in the tropical atmosphere over an ocean surface with cirrus present . . . 54

IB Measurements of sea surface albedo for diffuse radiation . 62

2B Fractions of direct and diffuse energy reaching the surface with cirrus clouds present and under cloudless

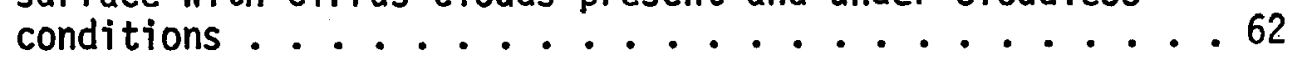




\section{LIST OF FIGURES}

$\underline{\text { Figure }}$

Page

1 Intensity of radiation transmitted through a model

cirrus cloud for a unit incident flux normal to the

cloud as a function of nadir angle ......... . 5

2 Extinction of shortwave energy by cloud layer vs.

ice concentration of cloud ............ 8

3 Probability of occurrence of single $\left(P_{1}\right)$, double $\left(P_{2}\right)$

and triple $\left(P_{3}\right)$, scattering as a function of optical

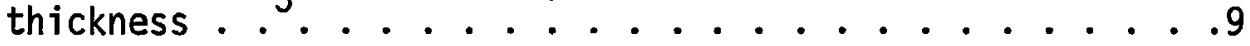

4 Cloud reflectivity as a function of solar zenith angle. . .12

5 Average transmission of cirrus clouds for shortwave radiation as a function of solar zenith angle .......13

6 Longwave emissivity of cirrus clouds as a function of geometric thickness ............ 16

7 Shortwave radiative temperature change in a $2 \mathrm{~km}$. thick cirrus cloud with a base pressure of $200 \mathrm{mb}$. as a function of cloud optical thickness . . . . . . . 20

8 Upward and downward shortwave irradiances at the

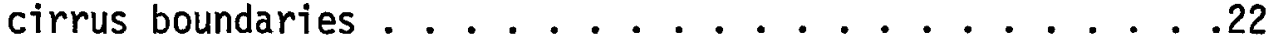

9 Upward and downward 1ongwave irradiances at the cirrus cloud boundaries............. 24

10 Contributions to the upward infrared irradiance at the cloud top and the downward infrared irradiance at cloud base from transmission and self-emission

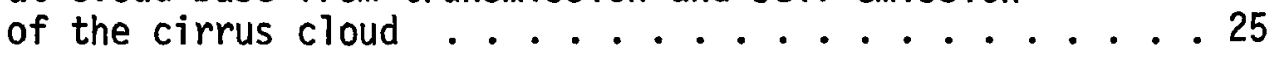

11 Divergence of net radiation in the surface to cloud base layer as a function of cirrus radiative properties. . 28

12 Reinterpretation of Figure 11 to show those combinations of cirrus radiative properties that will give the equivalent clear sky cooling in the subcloud layer . . 29

13 Divergence of net radiation in the cloud layer itself as a function of cirrus radiative properties . . . . . 30

14 The divergence of net radiation across cirrus clouds of different geometric thicknesses and base pressures for realistic ranges of $\varepsilon^{\star} ; 1-\omega_{0}=10^{-3} \ldots \ldots 33$ 


\section{LIST OF FIGURES - Continued}

Figure

$\underline{\text { Page }}$

15 Divergence of net radiation above the cloud layer as a function of cirrus radiative properties . . . . . 35

16 Vertical profiles of daily averaged radiational temperature change for a cloudless tropical atmosphere and for the same atmosphere with a cirrus cloud . . 36

17 Maximum and minimum radiative temperature change for three layers of the atmosphere as a function of cloud base location; $H=1 \mathrm{~km} . . . . . . . . .40$

18 Maximum and minimum radiative temperature change for three layers of the atmosphere as a function of cloud base location; $H=2 \mathrm{~km} . \ldots . . . \ldots . . . .41$

19 Net shortwave irradiance at the sea surface vs. solar zenith angle for the cloud free atmosphere and for three different cirrus clouds . . . . . . . . . 43

20 Net shortwave energy at the sea surface and at the top of the atmosphere for different cloud optical thicknesses ................... . . . . . . .

21 Shortwave energy absorbed by the atmosphere for different cloud optical thicknesses . . . . . . . . 46

22 Net radiative energy at the sea surface as a function of $\tau^{*}$ for three values of the sea surface temperature . . .48

23 Net radiative energy at the top of the atmosphere as a function of $\tau^{*}$ and $\varepsilon^{*}$ of the cirrus cloud ........ . 50

24a Shortwave and longwave daily radiative energy budgets for the cloudless atmosphere and with cirrus clouds

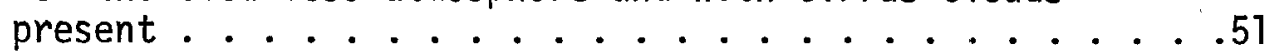

24b Total radiative energy, layer net energy change and radiative temperature change for cloudless and cirrus conditions ................... 52

lb Albedo of the sea surface for global radiation vs.

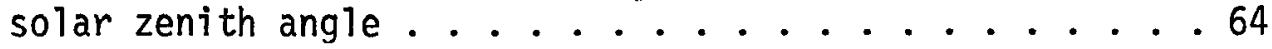




\section{LIST OF SYMBOLS}

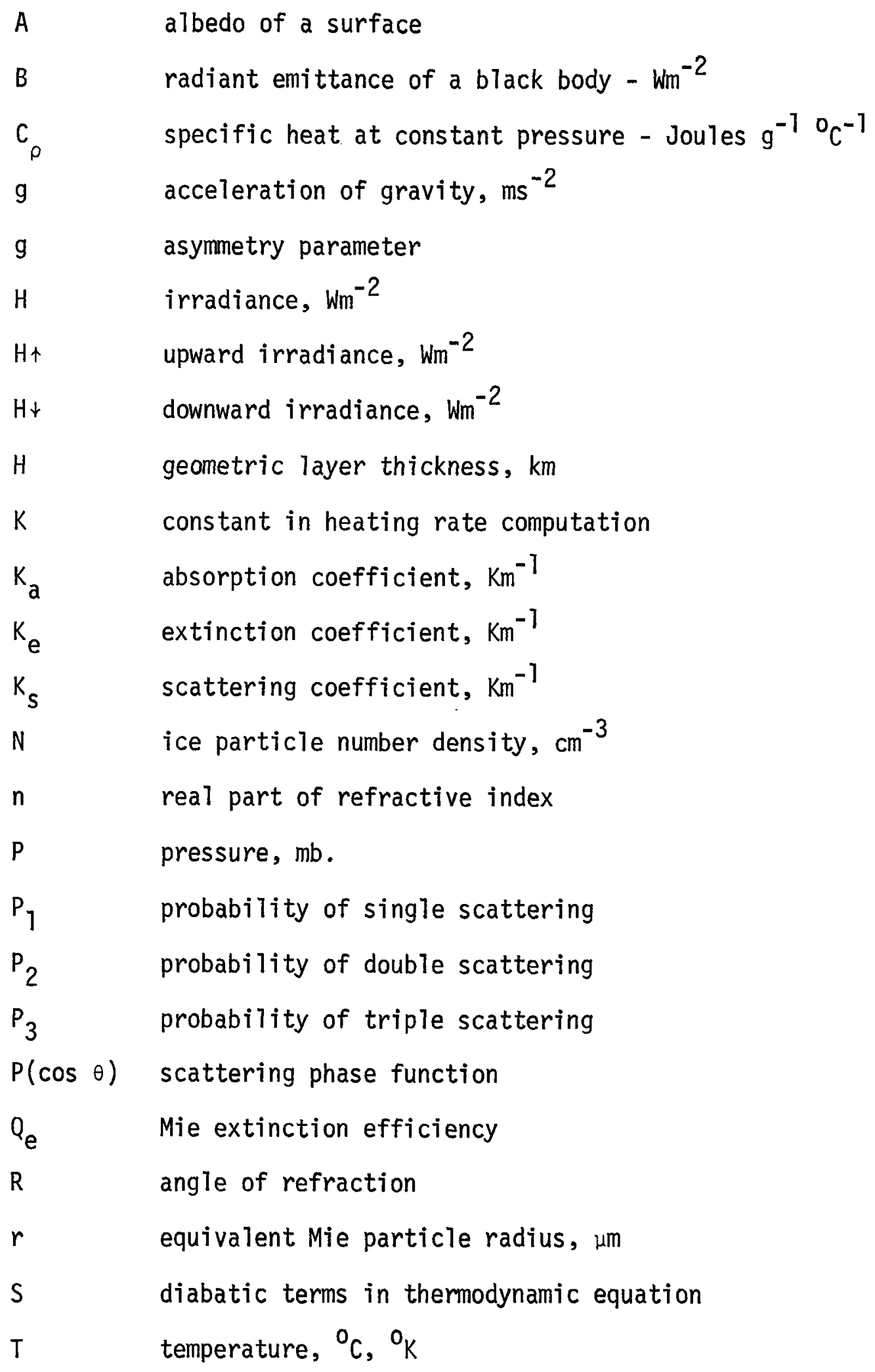




\section{LIST OF SYMBOLS - Continued}

$T$

t

$\bar{u}$

W

$\bar{W}$

$x$

Z

$\alpha$

$\gamma$

$\gamma d$

$\Delta$

$\varepsilon^{*}$

$\varepsilon^{\star \uparrow}$

$\varepsilon^{*} \downarrow$

$\varepsilon_{H}$

$\varepsilon_{Z}$

$\theta$

$\lambda$

$\rho \mathbf{i}$

p

$\sigma$

$\tau$

$\tau *$

$\tau * a$

$\tau * S$

$\omega_{0}$

transmissivity

time

eddy component of the horizontal wind, $\mathrm{m} \mathrm{sec}^{-1}$

cirrus cloud ice concentration, $\mathrm{g} \mathrm{m}^{-3}$

vertical velocity, $\mathrm{cm} \mathrm{sec}^{-1}$

horizontal cartesian coordinate

vertical cartesian coordinate

absorptivity

environmental lapse rate of temperature, ${ }^{0} \mathrm{C} \mathrm{km}-1$

dry adiabatic lapse rate of temperature, ${ }^{0} \mathrm{C} \mathrm{km}^{-1}$

finite difference operator

broad band effective emissivity

upward effective emissivity

downward effective emissivity

hemispherical emissivity

zenith emissivity

solar zenith angle

wavelength, $\mu \mathrm{m}$

density of ice, $\mathrm{gm} \mathrm{cm}^{-3}$

reflectivity

Stefan - Boltzman constant, $\mathrm{Wm}^{-2} \mathrm{o}_{K^{-4}}$

optical thickness

effective optical thickness

absorption optical thickness

scattering optical thickness

albedo of single scatter 
SUBSCRIPTS

$\begin{array}{ll}\text { B } & \text { Base } \\ \text { eq } & \text { equivalent } \\ \text { LW } & \text { longwave } \\ n & \text { net } \\ \text { S } & \text { surface } \\ \text { SFC } & \text { surface } \\ \text { SW } & \text { shortwave } \\ \text { T } & \text { top }\end{array}$




\section{INTRODUCTION}

Cirrus clouds are a regular feature of the atmosphere in the tropics. While their radiative properties are not particularly striking when compared to other clouds, their persistence in time and space make them an important feature in the study of the vertical transport of energy by radiation (Rieh1, 1962).

Clouds are the principal modulators of radiation in the tropical atmosphere. Investigation of the effects of cirrus clouds on the vertical profile of radiative divergence will yield insight into the energetics of the tropical atmosphere for atmospheric motions ranging from daily convective activity to the scale of the general circulation. Understanding of the energetics will aid in the development of a comprehensive theory of the tropical atmosphere.

The transfer of radiation through the tropical atmosphere in the presence of a cirrus cloud layer has been modelled in terms of two bulk radiative properties of the cloud: shortwave optical thickness, $\tau^{*}$, and broad-band infrared emissivity, $\varepsilon^{*}$. The optical thickness has been derived from the geometric thickness of the cloud layer by assuming the scattering phase function of the cirrus crystals and the concentration of ice in the cloud. The range of cirrus cloud effective infrared emissivity used was reported as a function of cloud geometric thickness by Kuhn and Weickmann (1969) and by Davis (1970). The shortwave and longwave irradiances at the cloud boundaries are correctly specified with minimum computational time by employing the cloud's bulk radiative properties in the computations; no information is available, however, concerning the spectral characteristics or the angular distribution of the radiation field which interacts with the cloud. 
This study reports the development of a model to calculate vertical profiles of net irradiance in the tropical atmosphere with cirrus clouds present. The divergence of net irradiance has been investigated as a function of bulk radiative properties of the cloud layer and the resulting radiative temperature change has been parameterized for three Tayers encompassing the entire atmosphere. A time integration of the irradiance values was performed over one day to determine the net radiative energy budget of the tropical atmosphere over an ocean surface for cloudless conditions and with cirrus clouds present. 
II. PROPERTIES OF CIRRUS CLOUDS

The determination of the radiative effects of a cirrus cloud Tayer requires information about the particles comprising the cloud. The composition, size, shape, orientation and number density of the cloud particles determine their effect on the scattering and absorption of incident solar energy. The geometric thickness, when combined with these microphysical cloud properties, determines the shortwave optical thickness and the range of probable infrared emissivity of the cirrus Tayer.

\section{A. Observed Physical Properties}

Three methods of investigation are applicable in determining the nature of the ice crystals composing cirroform clouds: direct measurements in situ may be carried out by aircraft instrumented to collect cloud hydrometeors; (2) in the laboratory ice crystals may be grown and studied under controlled conditions of temperature and water saturation; (3) deduction of physical characteristics of the crystals may be made from visual optical phenomena associated with cirrus cloud layers. Jacobowitz (1970) has discussed at length the results of these methods of investigation. He finds that the crystals of a cirrus cloud may be approximated by hexagonal columns of ice with a mean major axis of $150 \mu \mathrm{m}$ and a mean minor axis of $60 \mu \mathrm{m}$. He shows that the orientation of the crysta1s in space may be assumed to be random for calculations of the scattering phase function. The observed number density is of the order of $0.5 \mathrm{~cm}^{-3}$. His solutions of ice concentration as a function of cloud thickness and height are used to determine shortwave optical thickness of the cloud layer. 
Calculations of the divergence of net irradiance through a layer are sensitive to the location of the layer in the assumed temperature and moisture profile. Cirrus cloud layers may range in thickness from very thin wisps to layers $5 \mathrm{~km}$. thick. Heights of cirrus cloud bases in the tropics range from 9.7 to $12.8 \mathrm{~km}$. The highest cloud top observed that Davis (1970) reported during the BOMEX experiment was $14.9 \mathrm{~km}$. Calculations of the divergence of net irradiance are performed in this study for moderately thick cirrus clouds of infinite horizontal extent, one to two $\mathrm{km}$ thick with the lowest base at $9.7 \mathrm{~km}$ and the highest top at $16.0 \mathrm{~km}$.

\section{B. Shortwave Radiative Properties}

Knowledge of the particles comprising a cirrus cloud allows a calculation of the intensity of light scattered from a crystal as a function of the scattering angle. Jacobowitz (1971) has computed the scattering phase function for hexagonal crystals. His calculations include the effects of diffraction, reflection and refraction of light by the crystals. The intensity of radiation transmitted through a model cirrus cloud for a unit incident flux normal to the cloud is illustrated in Figure 1 as a function of the nadir angle. For optically thin layers, the transmission function is given to high accuracy by the phase function for single scattering. The scattering is dominated by a strong peak centered at $\theta=0^{\circ}$ where the intensity of scattered radiation is five orders of magnitude larger than that scattered in any other direction. 


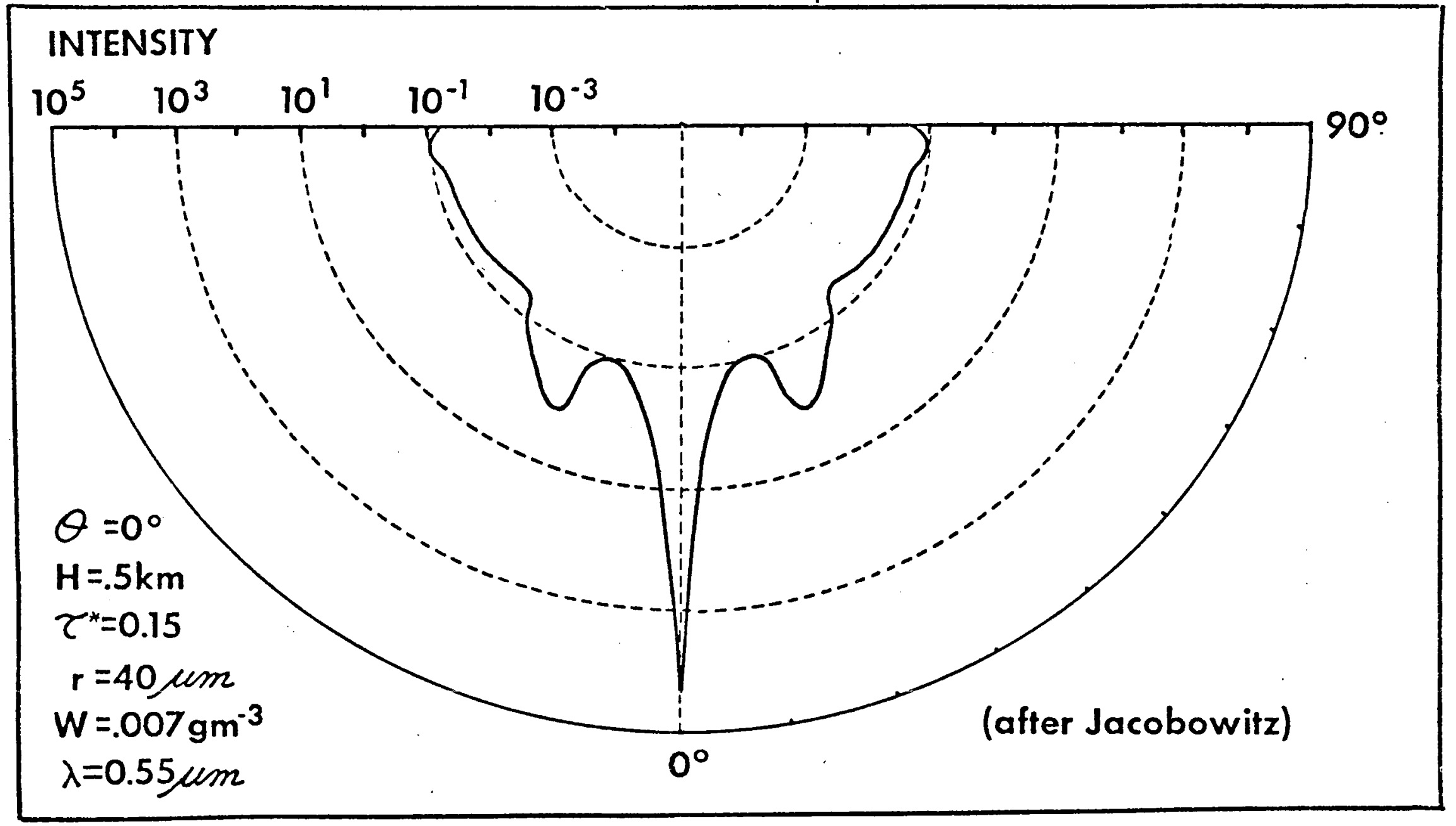

Figure 1. - Intensity of radiation transmitted through a model cirrus cloud for a unit incident flux normal to the cloud as a function of nadir angle. 
The effective optical thickness of a layer for monochromatic radiation is defined by

$$
\tau^{*}=\mathrm{K}_{\mathrm{e}} \cdot \mathrm{H}
$$

where $\mathrm{K}_{e}$ is the extinction coefficient and $H$ is the geometric thickness. Jacobowitz has determined that the scattering of light from cirrus crystals may be closely approximated by the scattering diagram computed from Mie theory for $40 \mu \mathrm{m}$ spheres. The extinction coefficient may then be written

$$
K_{e}=N \pi r^{2} Q_{e}
$$

where $N$ is the number density of cirrus crystals, $r$ is the equivalent Mie radius, and $Q_{e}$ the extinction efficiency. The ice concentration of the cloud is related to the number density by

$$
w=\frac{4}{3} \pi r^{3} \rho_{j} N
$$

where $\rho_{i}$ is the density of ice. Using these definitions, the expression for optical depth becomes

$$
\tau^{*}=\frac{3}{4} \frac{W H}{\rho_{i} r} Q_{e} \text {. }
$$

For particles large compared to the wavelength of light, $Q_{e} \simeq 2$ (Van de Hulst, 1957). For crystals of $40 \mu \mathrm{m}$ radius and with $\rho_{i}=0.92 \mathrm{~g} \mathrm{~cm}^{-3}$ equation (4) becomes

$$
\tau^{*} \simeq 40.7 \mathrm{WH}
$$

where $w$ has units $\mathrm{g} \mathrm{m}^{-3}$ and $H$ is in $\mathrm{km}$. $w$ and $H$ may vary over wide limits; the product of the two does not. The combinations of ice concentration $\mathrm{w}$ and geometric thickness $H$ used in the determination of optical thickness of cirrus clouds were taken from cloud solutions presented by Jacobowitz (1970). He inverted the problem of 
radiative transfer to solve for cloud properties using radiance data from the Nimbus III satellite infrared spectrometer. For example, a $2 \mathrm{~km}$ thick cloud with a base pressure of $200 \mathrm{mb}$ has an ice concentration ranging from one to three $\mathrm{mg} \mathrm{m}^{-3}$. From equation (5) this gives a range of $\tau^{*}$ from 0.0814 to 0.2442 . Figure 2 shows shortwave extinction vs. ice concentration $w$ for a $2 \mathrm{~km}$ thick cloud with a base pressure of $200 \mathrm{mb}$.

These small values of the optical thickness indicate that the radiation that interacts with the cloud particles is scattered only once. Figure 3 shows the probability of occurrence of single $\left(P_{1}\right)$, double $\left(P_{2}\right)$, and triple $\left(P_{3}\right)$ scattering determined by Bugnolo (1960) as a function of optical thickness. For $\tau^{\star}=0.2442$, the probability of double scattering events is less than $3 \%$ and the probability of a triple scatter is less than $1 \%$. Therefore, the solar irradiance at the base of the cloud will consist primarily of energy directly transmitted through the cloud and energy scattered into the forward direction after interaction with one cirrus crystal. The irradiance reflected by a cirrus cloud is also primarily the result of interactions with single crystals. Because the individual crystals determine the surface of the cloud, small horizontal irregularities in the cloud layer will have virtually no effect on the reflected light leaving the cirrus cloud.

The effects of absorption of solar radiation by cirrus ice crystals were derived from estimates of the single scattering albedo $\omega_{0}$ given by Danielson et a], (1969). The albedo of single scattering is defined as the ratio of the amount of light scattered by a layer to that which is scattered and absorbed. 


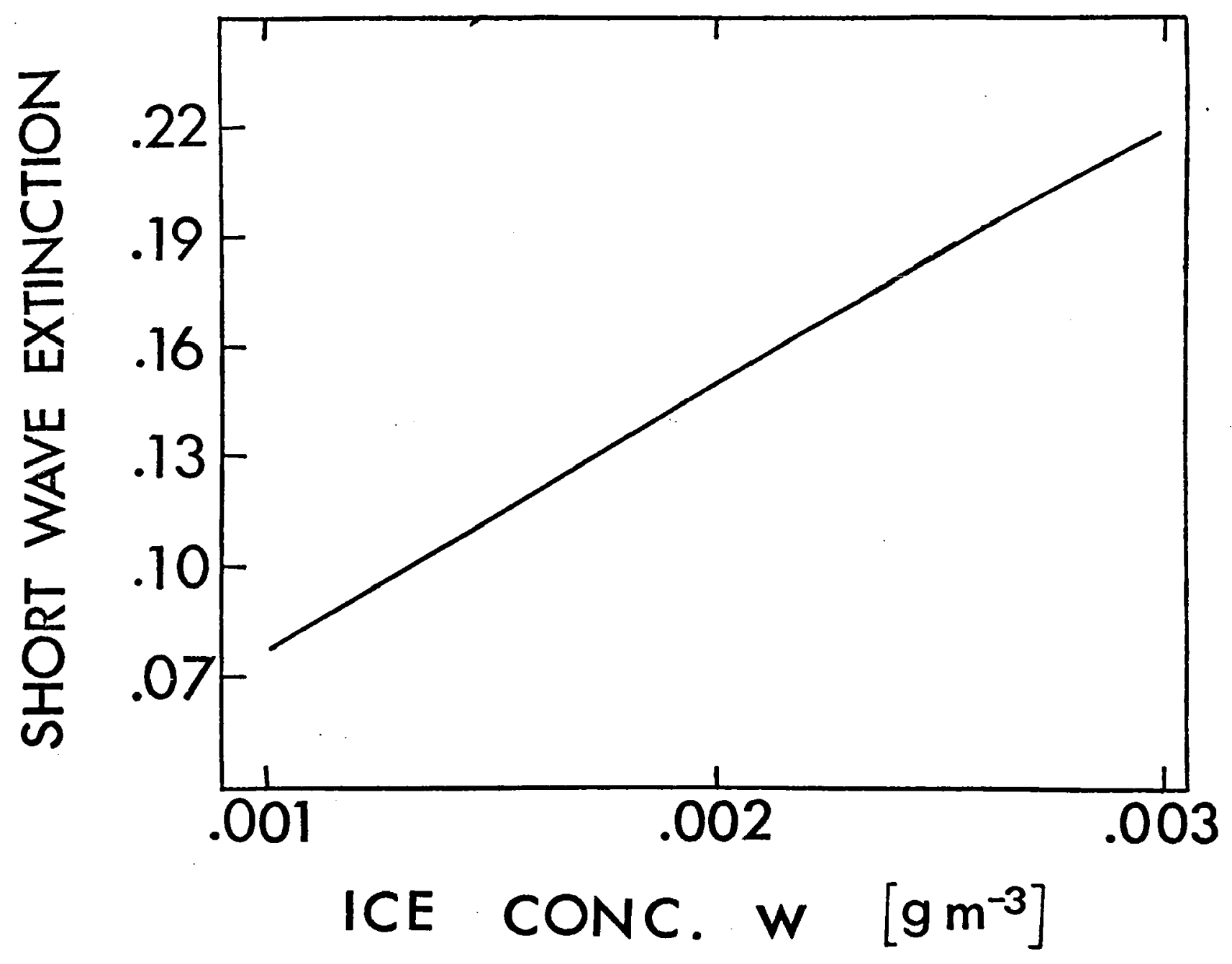

Figure 2. - Extinction of shortwave energy by cloud layer vs. ice concentration of cloud; $H=2 \mathrm{~km}, \mathrm{P}_{B}=200 \mathrm{mb}$. 


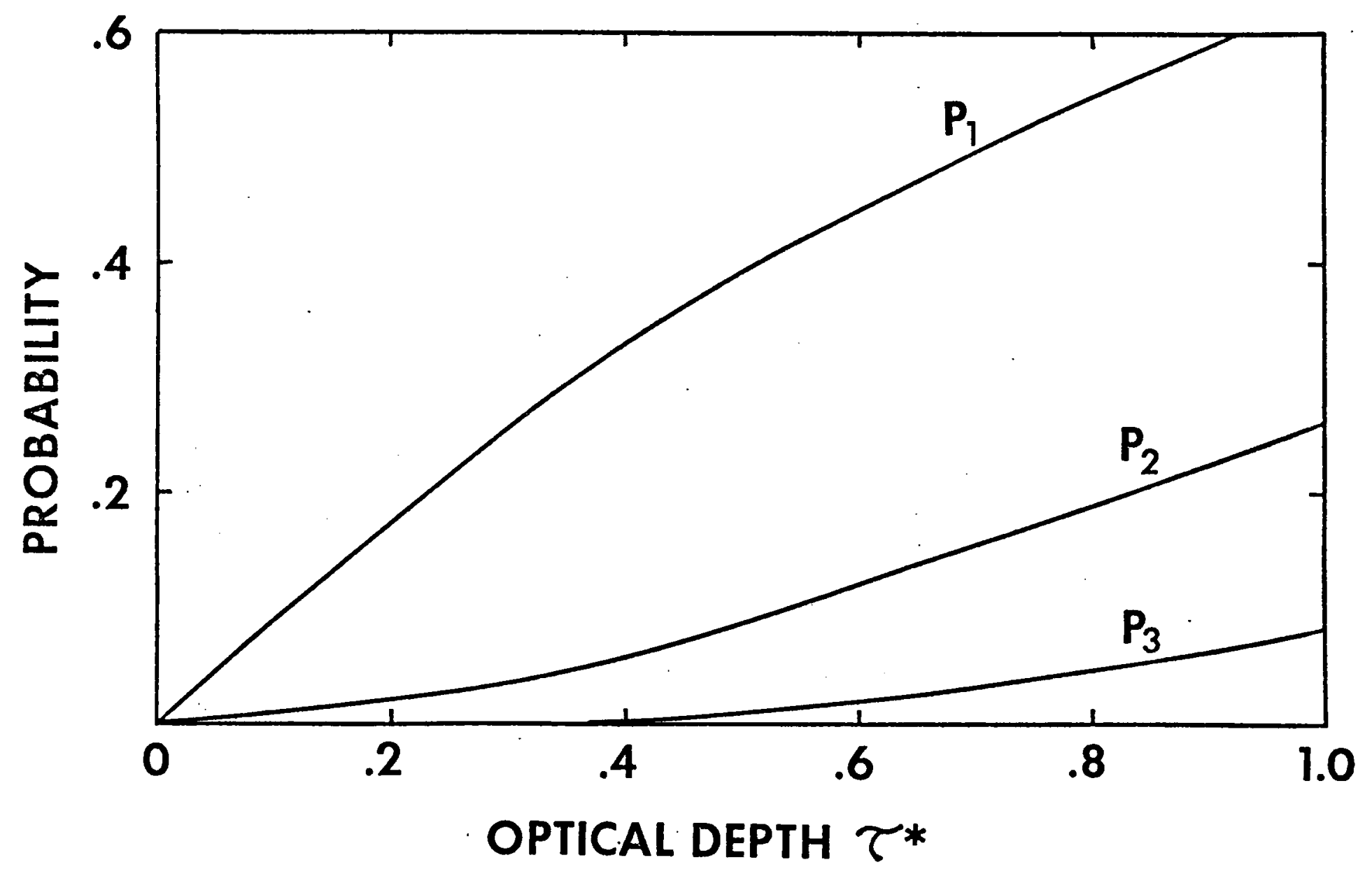

Figure 3. - Probability of occurrence of single $\left(P_{1}\right)$, double $\left(P_{2}\right)$, and triple $\left(\mathrm{P}_{3}\right)$, scattering as a function of optical thickness. 


$$
\omega_{0}=\frac{k_{s}}{k_{s}+k_{a}}
$$

where $K_{s}$ is the scattering coefficient and $k_{a}$ is the absorption coefficient and $K_{s}+K_{a}=K_{e}$. Danielson estimates $1-\omega_{0} \simeq 10^{-3}$ for terrestrial clouds. This gives

$$
\begin{gathered}
1-\omega_{0}=1-\frac{K_{s}}{K_{e}}=\frac{K_{a}}{K_{e}}=10^{-3} \\
\omega_{0}=\frac{K_{s}}{K_{e}}=0.999 .
\end{gathered}
$$

Using equation (1), an absorption optical thickness $\tau^{*}{ }_{a}$ and $a$ scattering optical thickness $\tau^{*}{ }_{S}$ may be defined:

$$
\tau^{*}=\tau_{a}^{*}+\tau_{s}^{*}=\left(K_{a}+K_{s}\right) H
$$

and

$$
\begin{gathered}
\tau_{a}^{*}=\left(1-\omega_{0}\right) \tau^{*} \\
\tau^{*}{ }_{s}=\omega_{0} \tau^{*} .
\end{gathered}
$$

The transmissivity of a layer of optical thickness $\tau^{*}$ is

$$
T=\exp \left(-\tau^{*} \sec \theta\right)
$$

where $\theta$ is the solar zenith angle and the values of $\sec \theta$ are reduced by atmospheric refraction and curvature of the earth's atmosphere. The extinction of solar radiation by the cloud layer is given at each solar zenith angle by 1.-T. Reflectivity $p$ and absorptivity $\alpha$ are expressed in terms of the scattering and absorption optical thicknesses by

$$
\begin{aligned}
& \rho=1 \cdot-\exp \left(-\tau^{*} \sec \theta\right) \\
& \alpha=1-\exp \left(-\tau^{*}{ }_{a} \sec \theta\right)
\end{aligned}
$$


In order to compare the results of these calculations to solutions of the transfer equation employing the scattering phase function in its explicit form, it is necessary to scale the optical thickness derived from exact theory (Van de Hulst \& Grossman, 1968).

$$
\tau^{*}=(1-g) \tau
$$

where $\tau^{*}$ is the effective optical thickness one would assume in an isotropic scattering problem in order to obtain computed irradiance values similar to those computed for thickness $\tau$ in an anisotropic problem. The asymmetry parameter, $g$, is defined by

$$
g=\frac{1}{2} \int_{-1}^{+1} P(\cos \theta) \cos \theta d(\cos \theta)
$$

where $g$ is the weighted mean over the sphere of the cosine of the scattering angle with the phase function $\mathrm{P}(\cos \theta)$ as a weighting function; $g$ ranges from +1 for total forward scattering to -1 for total back scatter. Figure 4 shows theoretical curves computed by Liou (1973) and Hansen (1969) of cloud reflectivity as a function of solar zenith angle for $\tau=2$ and $\tau=.5$ (broken lines); and the reflectivity employed in the present study for $\tau^{*}=0.1628$ and $\tau^{*}=0.0452$ (solid lines). The curves are in approximate coincidence for an asymmetry parameter $g=0.9$. Danielson et al (1969) estimates $\mathrm{g}=0.875$ for terrestrial clouds. The average cirrus cloud total albedo of $20 \%$ with solar zenith angle $25^{\circ}$ measured by Drummond and Hickey (1971) is plotted in Figure 4 for comparison.

Observations of the transmissivity of cirrus clouds at visible wavelengths have been tabulated by Kondratyev (1969) and are illustrated in Figure 5 . The number below each point represents 


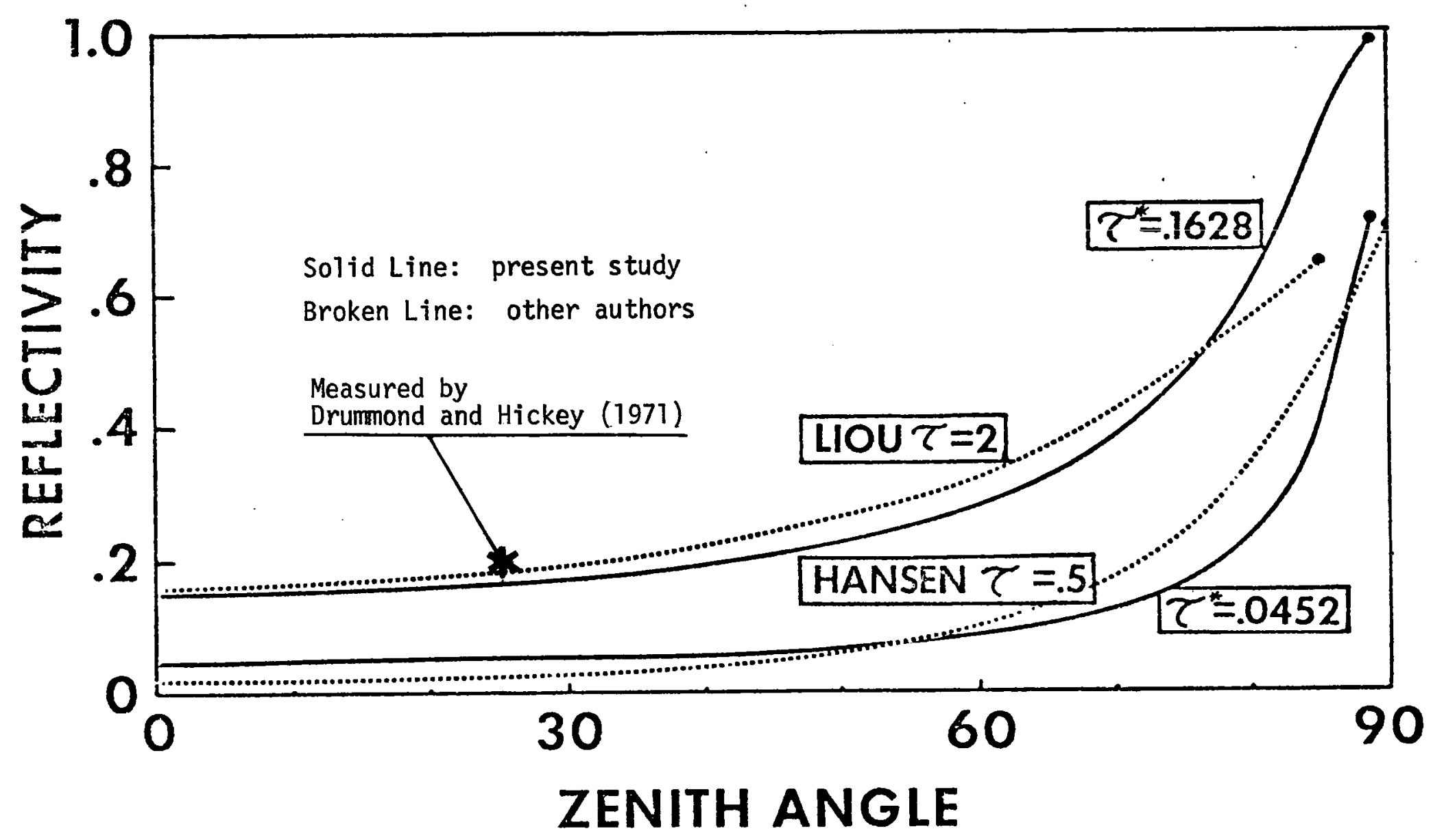

Figure 4. - Cloud reflectivity as a function of solar zenith angle. 


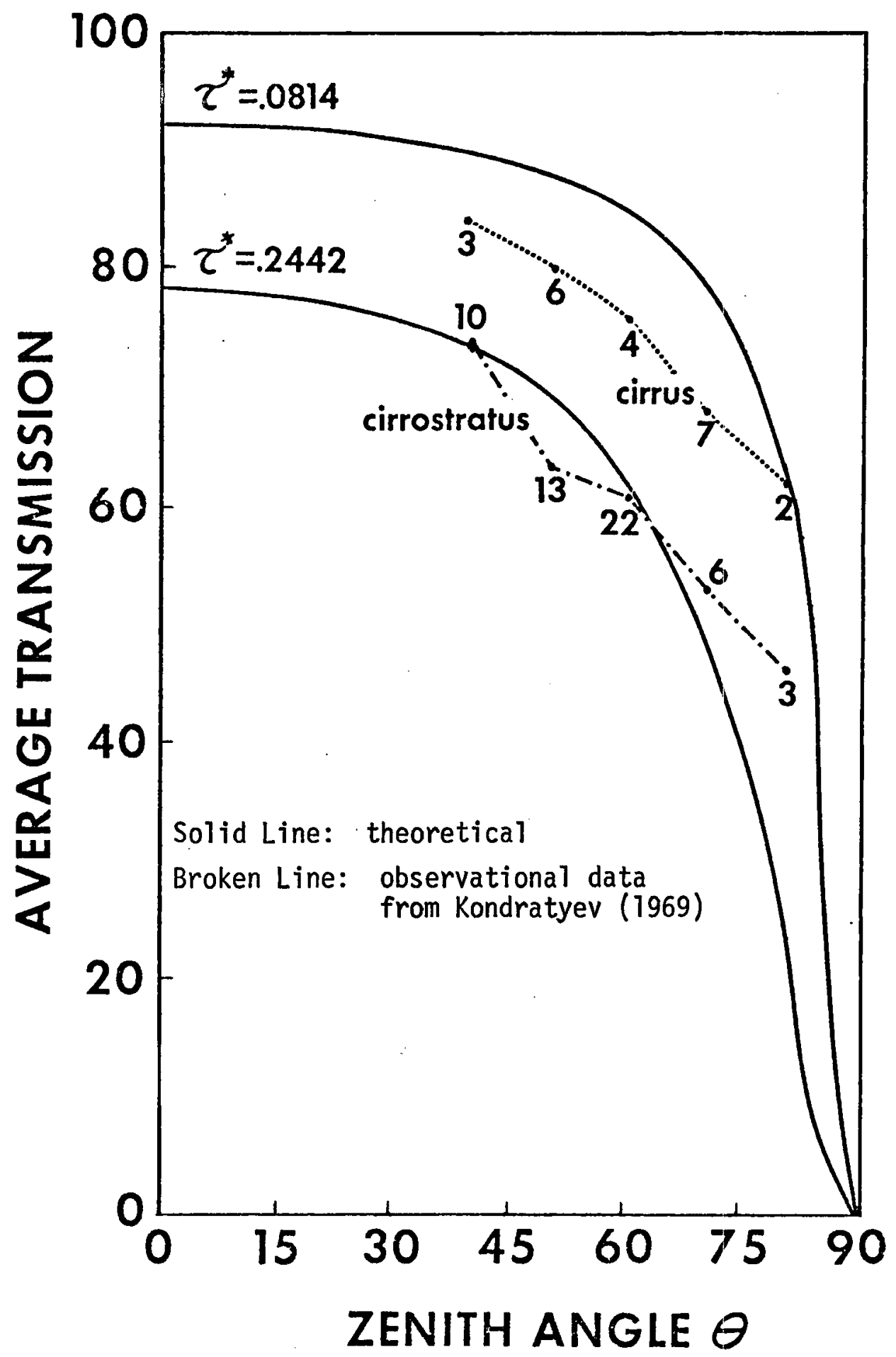

Figure 5. - Average transmission of cirrus clouds for shortwave radiation as a function of solar zenith angle. 
the number of abservations at that zenith angle included in the average. Measured deviations of $30-40 \%$ in the average transmission are included in the averages. The theoretical curves for a $2 \mathrm{~km}$ thick cloud at $200 \mathrm{mb}$. with two ice concentrations of .001 and .003 $\mathrm{g} \mathrm{m}^{-3}$ are shown for comparison.

\section{Longwave Radiative Properties}

The transfer of infrared radiation through a cloud layer may be modelled in terms of the broad-band layer emissivity (c.f. section III B.). Determinations of infrared emissivities of cirrus clouds as a function of geometric thickness have been made by Cox (1969), Kuhn and Weickmann (1969), and by Davis (1970). The effective emissivity (Gergen, 1958) is determined from the equivalent radiation at cloud top and cloud base by

$$
\varepsilon^{*}=\frac{H_{e q}(2)-H_{e q}(1)}{B_{e q}(2)-B_{e q}(1)}
$$

where $H_{e q}(1)$ is the equivalent radiation at cloud top, defined as the upward irradiance plus the downward irradiance divided by two. $B_{\text {eq }}(1)$ is the corresponding equivalent irradiance from a black body radiating at the temperature of the cloud top. The subscript (2) refers to cloud base. The emissivity derived in this manner implicitly includes the effects of emission by gases within the cloud layer and any scattering by the cloud hydrometeors.

An upward effective emissivity $\left(\varepsilon^{*} \uparrow\right)$ and a downward effective emissivity $\left(\varepsilon^{*} \downarrow\right)$ may be defined from observations of the divergence of the upward and downward longwave irradiances. According to Cox (1969), it is normally found that $\varepsilon^{*} \downarrow>\varepsilon^{*}>\varepsilon^{*} \uparrow$. Because the 
longwave radiative transfer scheme treats the above cloud layers independently from the below cloud layers, the radiative effects of cirrus cloud found to have $\varepsilon^{\star_{\downarrow}} \neq \varepsilon^{*}$. will be derived in the present study from a composite of cases involving two different values of $\varepsilon^{*}$.

Figure 6 illustrates the results of measurements by Kuhn and Weickmann and by Davis and calculations performed for discrete wave numbers by Jacobowitz (1970) of emissivity as a function of cloud geometric thickness. The zenith emissivity $\varepsilon_{z}=1 .-\exp (-\tau)$ measured by Kuhn and Weickmann was transformed to hemispherical emissivity $\varepsilon_{H}$ by integrating $\varepsilon_{z}$ over the hemisphere.

Because of the large variations in observed emissivities and the poor relationship of $\varepsilon^{*}$ to $H$, radiative transfer calculations were performed for several geometric thicknesses and for a range of emissivity values specified in Table 2, section IV.

A summary of the geometric, physical and radiative parameters which are considered in deriving the radiative effects of cirrus cloud is presented in Table 1. 


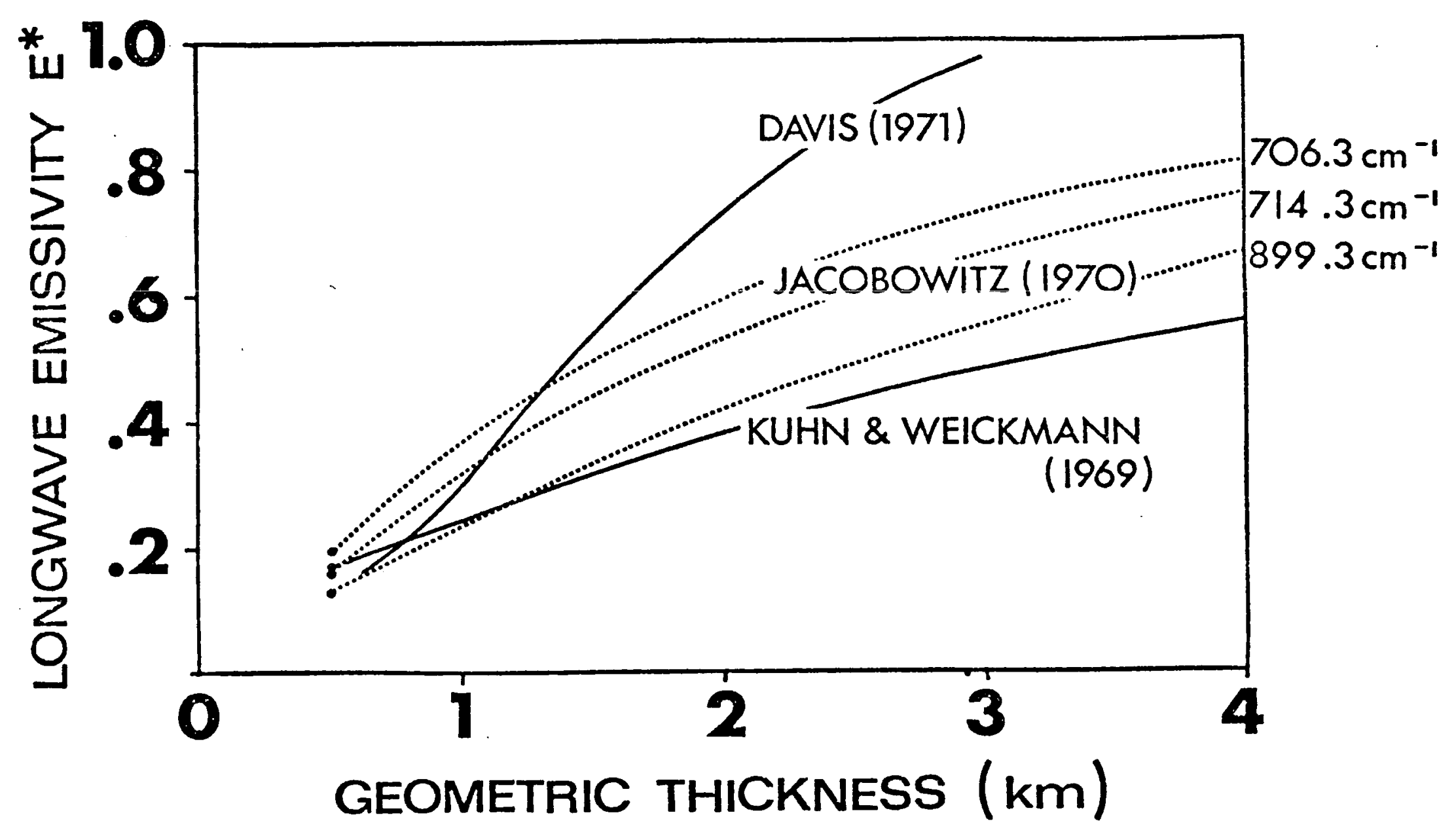

Figure 6. - Longwave emissivity of cirrus clouds as a function of geometric thickness. 
TABLE 1.

GEOMETRY

PHYSICAL PROPERTIES

CLOUD

RADIATIVE PROPERTIES

DERIVED PROPERTIES

DAY OF YEAR

LATITUDE

SOLAR ZENITH ANGLE $\theta$
EFFECTIVE DROPLET RADIUS $r$ ICE CONCENTRATION W

CLOUD BASE PRESSURE $P_{B}$ CLOUD THICKNESS $\mathrm{H}$ ATMOSPHERIC PROFILE

$P, T, \mathrm{H}_{2} \mathrm{O}, \mathrm{CO}_{2}, \mathrm{O}_{3}$
S. W. OPTICAL DEPTH $\tau^{*}$

L. W. EMISSIVITY $\varepsilon^{*}$
IRRADIANCE PROFILES DIVERGENCE OF NET IRRADIANCE PARAMETERIZED HEATING RATES RADIATIVE ENERGY BUDGET 
III. THE CLOUD MODEL.

In this study, shortwave and longwave irradiances are computed separately by numerical radiative transfer techniques for mean tropical temperature and moisture profiles. The shortwave transfer technique treats absorption of solar radiation by water vapor, carbon dioxide and ozone; scattering by molecules in the cloud free atmosphere; and reflection, absorption and transmission of shortwave radiation by the cloud layer. The short wavelength region considered is from $0.3 \mu \mathrm{m}$ to $3.0 \mu \mathrm{m}$. The longwave radiative transfer technique treats emission by the same three gaseous constituents of the atmosphere and transmission and emission by the cloud layer. The Tongwave spectrum is confined to the interval $3.0 \mu \mathrm{m}$ to $55.0 \mu \mathrm{m}$ in wavelength.

\section{A. Shortwave Radiative Transfer}

The transfer of solar radiation through the atmosphere was calculated using the absorption data for water vapor, carbon dioxide and ozone given as a function of absorber amount by Manabe and Moller (1961). Temperature, mixing ratio of water vapor and ozone taken from the United States Standard Atmosphere Supplements (1966) for $15^{\circ} \mathrm{N}$ latitude were specified at each pressure level. The amount of carbon dioxide was assumed constant as a function of height at 0.0447 percent by weight. The total radiative temperature change for each layer is the sum of the temperature changes due to absorption of radiation by the three gases. Any overlap between absorption bands of the different gases was ignored. The altitude of the sun above the horizon is specified uniquely by the day of the year, latitude and time of day. Instantaneous shortwave 
heating rates, computed for 23 time steps from surrise to local noon were summed and multiplied by two to give the total shortwave heating through the day.

The addition of a cirrus cloud to the atmosphere requires that solar radiation reflected and absorbed by ice crystals in the cloud layer be considered in the transfer computation. At each time step, the albedo of the cirrus layer is given by equation (13). The reflected irradiance $\mathrm{H} \uparrow$ is equat to $p \cdot H_{\psi}$. The cirrus layer was assumed to be a diffuse reflector and the optical thickness of each layer of the atmosphere above the cloud was scaled by the diffusivity factor 1.66 , formed by a hemispherical integration of optical thickness over solid angle, (Goody, 1964), for computing absorption of the reflected irradiance. Above the cloud the downward flux at each level was given by the sum of the direct beam irradiance and the irradiance reflected by the cloud which was rescattered by molecules into the lower hemisphere. The upward flux above the cloud is the sum of irradiance directly reflected by the cloud and that which is scattered upward by molecular constituents above the cloud. The absorption of radiation by ice crystals is computed from equation (14). The total heating of the cloud layer is then given by the sum of the heating caused by gaseous absorption and absorption by cirrus crystals.

$$
\left(\frac{\Delta T}{\Delta t}\right)_{S W}^{\text {TOTAL }}=\left(\frac{\Delta T}{\Delta t}\right)_{S W}^{\text {GASEOUS }}+\left(\frac{\Delta T}{\Delta t}\right)_{S W}^{\text {ICE }}
$$

The shortwave radiative temperature change in a cirrus cloud layer $2 \mathrm{~km}$ thick with a base pressure of $200 \mathrm{mb}$ is illustrated in Figure 7 as a function of optical thickness. The absorptivity of 


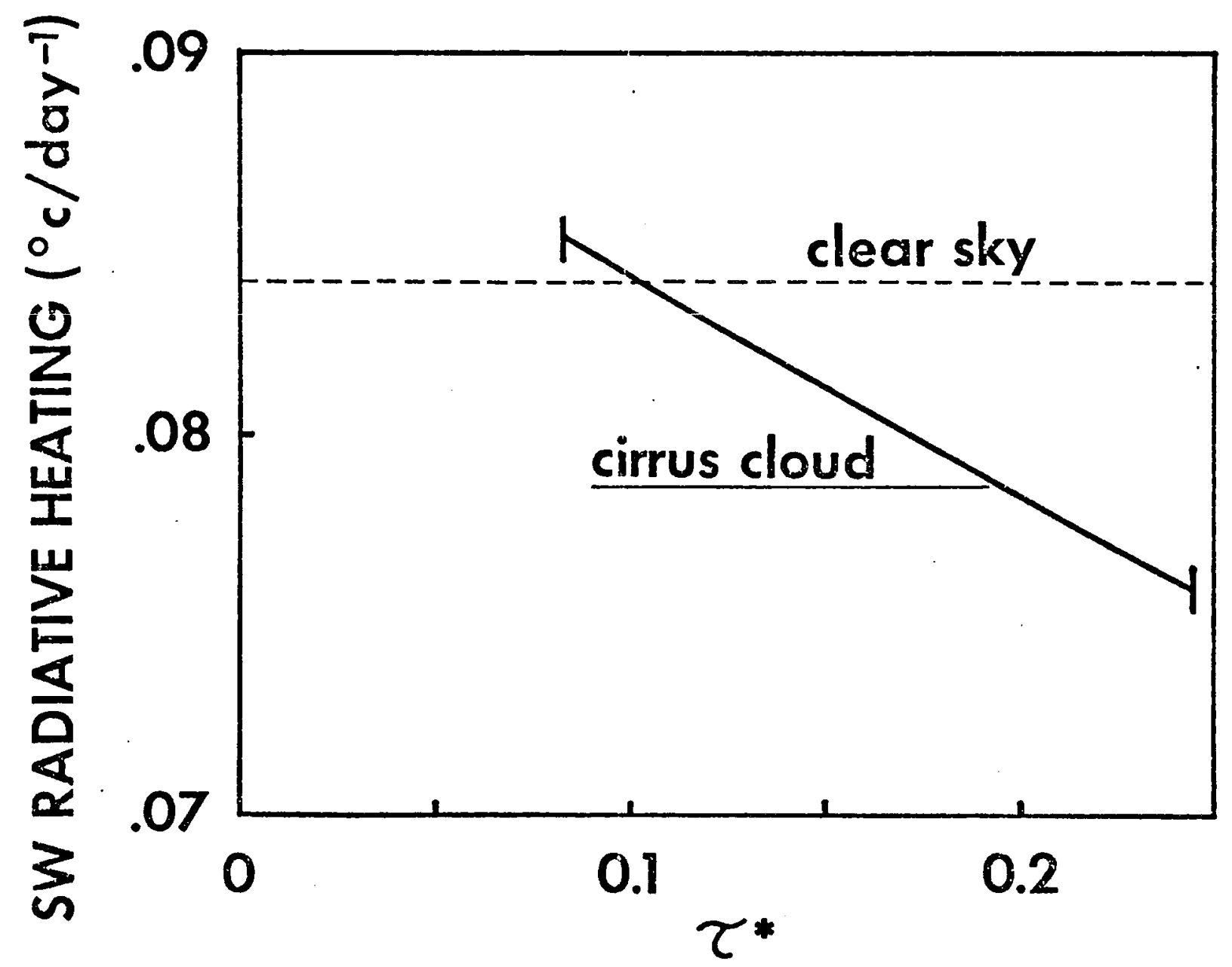

Figure 7. - Shortwave radiative temperature change in a $2 \mathrm{~km}$. thick cirrus cloud with base pressure of $200 \mathrm{mb}$. as a function of cloud optical thickness. 
the cirrus cloud increases with increasing $\tau^{*}$. The radiative heating of the cloud layer, however, is increased over the clear sky value only for smali values of optical thickness. For $T^{*}>0.7$, increased reflectivity of the cloud layer decreases the amount of solar energy available for absorption in the cloud.

Figure 8 illustrates the conditions imposed on the shortwave irradiance at the boundaries of a cirrus cloud. The upward and downward fluxes are defined by the optical thickness $\tau^{*}$, the albedo of single scatter, $\omega_{0}$, and the solar zenith angle.

Energy which is not reflected or absorbed by the cirrus cloud is transmitted to the layers of the atmosphere below the cloud. A surface albedo of zero is assumed in the initial computations; this constraint is relaxed in the discussion of radiative energy budgets. The resuits of the computations are most applicable to cirrus clouds located over the tropical oceans (c.f. Appendix B).

\section{B. Longwave Radiative Transfer}

The transfer of Tongwave radiation ( $3 \mu \mathrm{m}$ to $55 \mu \mathrm{m}$ ) was calculated with a numerical computation technique reported by Cox (1973). Temperature, moisture, and ozone profiles taken from the United States Standard Atmosphere Supplements (1966) for $15^{\circ} \mathrm{N}$ latitude were used in the calculations.

The radiative temperature change of a volume of the atmosphere is caused by the divergence of net radiation across that volume. Assuming horizontal homogeneous stratification and employing the hydrostatic assumption, the radiative temperature change may be written

$$
\frac{d T}{d t}=-\frac{g}{C_{p}} \frac{d H_{n}}{d P} .
$$




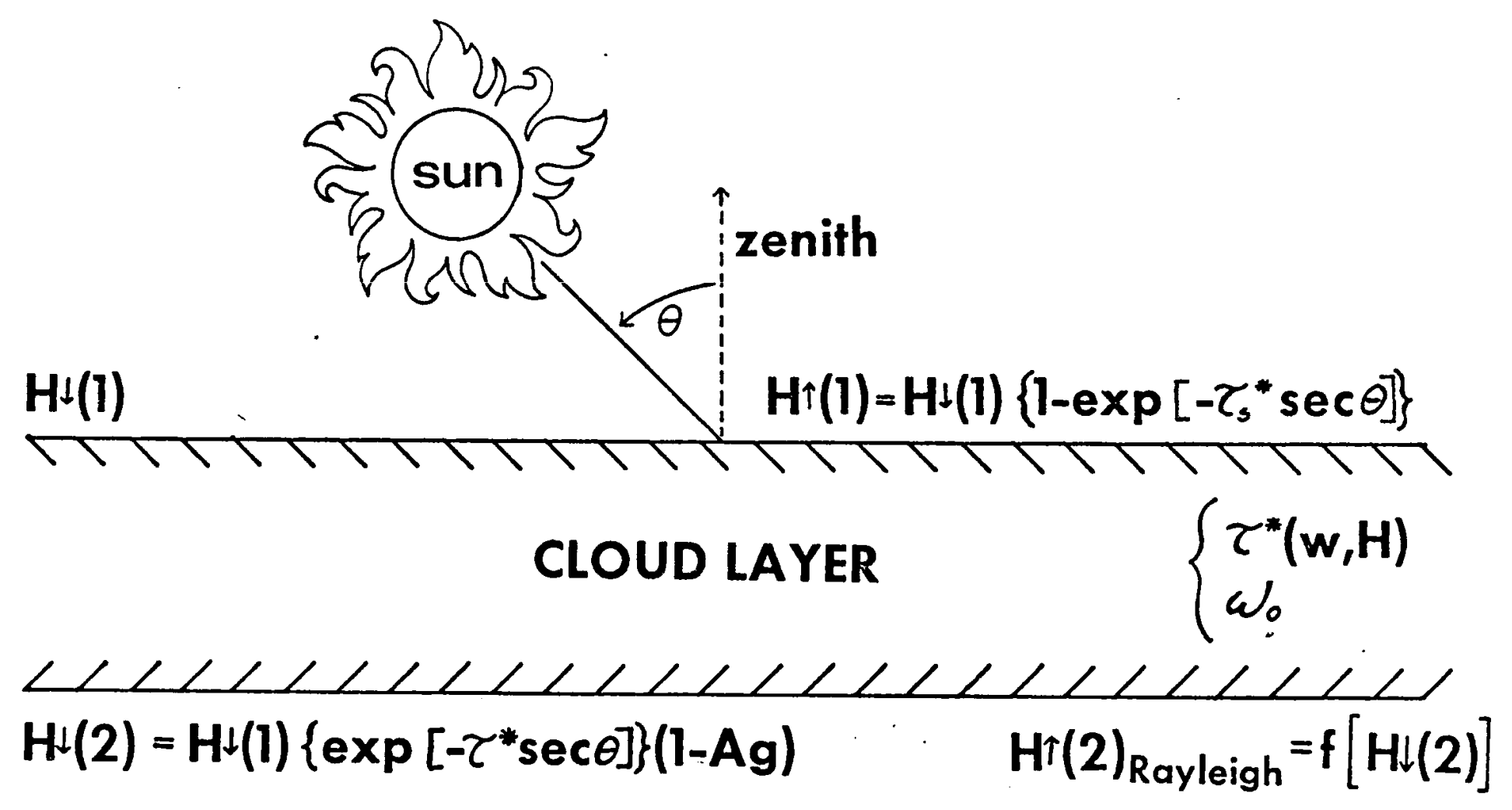

Figure 8. - Upward and downward shortwave irradiances at the cirrus boundaries. 
In finite difference form, this is computed as

$$
\frac{\Delta T}{\Delta t}=k \frac{H_{n}(2)-H_{n}(1)}{P(2)-P(1)}
$$

where $K=8.43^{\circ} \mathrm{Cday}^{-1} \mathrm{~W}^{-1} \mathrm{~m}^{2} \mathrm{mb}, H_{n}(1)=H_{\uparrow}(1)-H_{\downarrow}(1)$ is the net LW irradiance at the top of the layer in units of $\mathrm{Wm}^{-2}$ and $P(1)$ is pressure in mb. The subscript (2) refers to the base of the layer. The units of $\Delta T / \Delta t$ are ${ }^{\circ} \mathrm{C} d a y^{-1}$.

The longwave radiative effects of a cloud layer were specified by the broadband emissivity of the layer defined in equation (17). Figure 9 shows the conditions imposed on the longwave irradiance at the boundaries of a cirrus cloud. The downward irradiance at cloud top $H \downarrow(1)$ and the upward irradiance at cloud base $H_{\uparrow}(2)$ were assumed to be the same as the clear sky value. The contributions to the upward irradiance at cloud top $H_{\uparrow}(1)$ and the downward irradiance at cloud base $H_{\downarrow}(2)$ are illustrated in Figure 10 as a function of cloud emissivity $\varepsilon^{*}$. A $2 \mathrm{~km}$ thick cloud with a base pressure of $200 \mathrm{mb}$ was assumed for the calculations. The effects of transmission and self emission are given by the solid lines for $H+(1)$ and by the broken lines for $\mathrm{H} \downarrow(2)$. In each case, the dark $l$ ine is the sum of the two lighter ones. The result is a decrease of $H_{\uparrow}(1)$ and an increase of $\mathrm{H}_{\downarrow}(2)$ relative to the values calculated in the clear atmosphere. This causes an increased convergence of net irradiance both beneath the cloud and within the cloud layer itself. 


\section{LONGWAVE TRANSFER: BOUNDARY CONDITIONS}

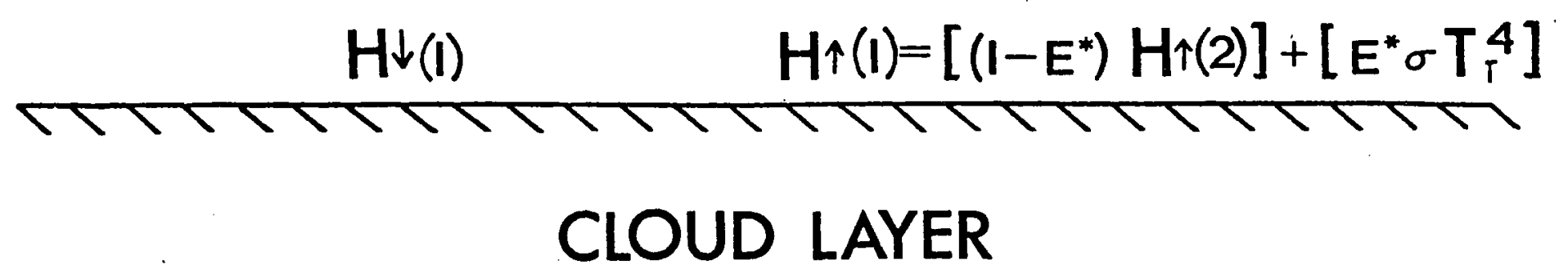

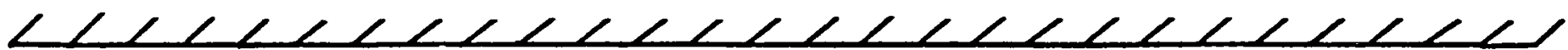

$H \downarrow(2)=\left[\left(1-E^{*}\right) H \downarrow(1)\right]+\left[E^{*} \sigma T_{B}^{4}\right]$

$\mathrm{H} \uparrow(2)$

Figure 9. - Upward and downward longwave irradiances at the cirrus cloud boundaries. 


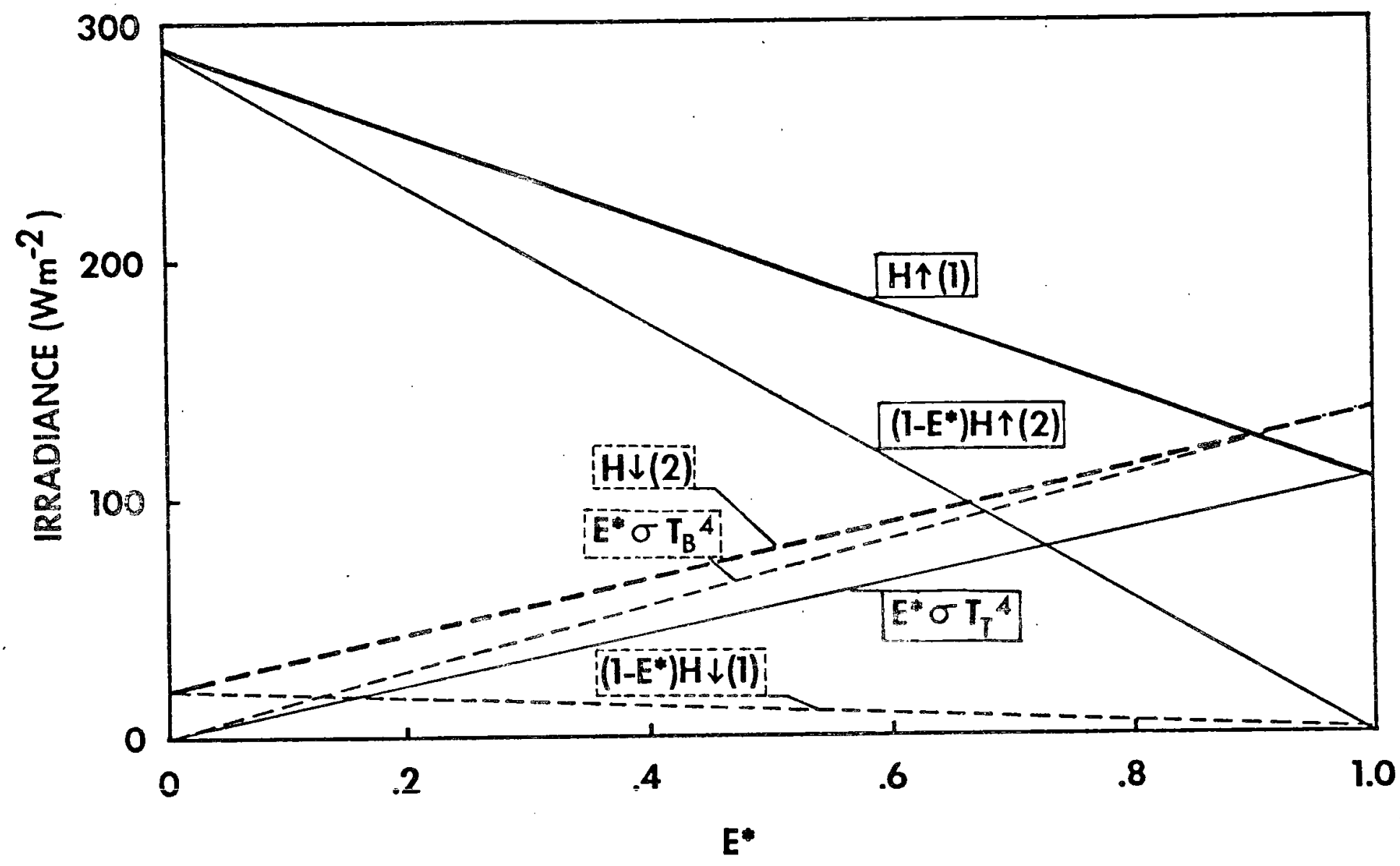

Figure 10. - Contributions to the upward infrared irradiance at the cloud top and the downward infrared irradiance at cloud base from transmission and self-emission of the cirrus cloud. 
IV. MODEL RESULTS

A. Radiative Divergence

Shortwave and longwave radiative divergence values were calculated for realistic values of optical thickness and longwave emissivity of the cirrus layer. Three cloud base pressures and two geometric thicknesses were considered. The United States Standard Tropical Atmosphere (1966) was used in the computations. Table 2 summarizes the cirrus cloud characteristics examined:

Table 2.

\begin{tabular}{cccc} 
PRESSURE OF CLOUD BASE & $\begin{array}{c}\text { GEOMETRIC THICKNESS } \\
(\mathrm{km} .)\end{array}$ & $\left\{\tau^{*}\right\}$ & $\left\{\varepsilon^{\star}\right\}$ \\
\hline 300 & 1 & $.2035-.2849$ & $.2-.4$ \\
300 & 2 & $.1221-.2442$ & $.3-.8$ \\
200 & 1 & $.0814-.2035$ & $.2-.4$ \\
200 & 2 & $.0814-.2442$ & $.3-.8$ \\
150 & 1 & $.0407-.1221$ & $.2-.4$ \\
150 & 2 & $.0407-.0814$ & $.3-.8$
\end{tabular}

The divergence of net radiation in the layer from surface to cloud base is shown in Figure 11 for a $2 \mathrm{~km}$. thick cirrus cloud with a base pressure of $200 \mathrm{mb}$. The computations were performed for latitude $15^{\circ} \mathrm{N}$ and the 183rd day of the year and for a realistic range of $\tau^{*}$ and $\varepsilon^{*}$. Divergence in the subcloud layer decreases with increasing cloud infrared emissivity. This is to be expected from the results of Figure 9 where the downward flux at cloud base increases with $\varepsilon^{*}$ while the upward flux remains constant. Divergence also decreases with decreasing shortwave optical thickness. 
Optically thin clouds transmit solar energy readily, further suppressing the cooling by allowing gaseous absorption of shortwave energy beneath the cloud. For the range of probable cirrus layer radiative characteristics, the divergence is less than that calculated without the cloud layer present. The clear sky value of $178 \mathrm{wm}^{-2}(800 \mathrm{mb})^{-1}$ is indicated by the horizontal line in Figure 11. This clear sky radiative temperature change is illustrated in Figure 12 as the locus of shortwave extinction and infrared emissivity which gives a radiative temperature change of $-1.9^{0} \mathrm{C} \mathrm{day}^{-1}$ through this subcloud layer. Cirrus clouds $2 \mathrm{~km}$ thick with a base pressure of $200 \mathrm{mb}$ have radiative properties lying completely in the region of suppressed cooling. The dependence of the below-cloud divergence on the sun's declination is very slight. Between the winter solstice (solar declination = $-23.5^{\circ}$ ) and day 115 (solar declination $=+15^{\circ}$ ), the daily cooling rate decreases by $0.1^{\circ} \mathrm{C} \mathrm{day}^{-1}$. This is a $6 \%$ variation of radiative temperature change over a large range of solar zenith angles.

The divergence of net radiation within the cloud layer itself is shown in Figure 13 for the same $2 \mathrm{~km}$ thick cirrus cloud. The values are negative implying a radiative warming of the cirrus layer. The longwave effects are dominant with divergence increasing with cloud blackness. This warming may be expressed (see Appendix A) as a linear function of the cloud longwave emissivity.

$$
\left(\frac{\Delta T}{\Delta t}\right)^{\text {TOTAL }}=\varepsilon^{*}\left(\frac{\Delta T}{\Delta t}\right)_{\text {BLACK }}^{L W}+\left(\frac{\Delta T}{\Delta t}\right)^{S W}
$$




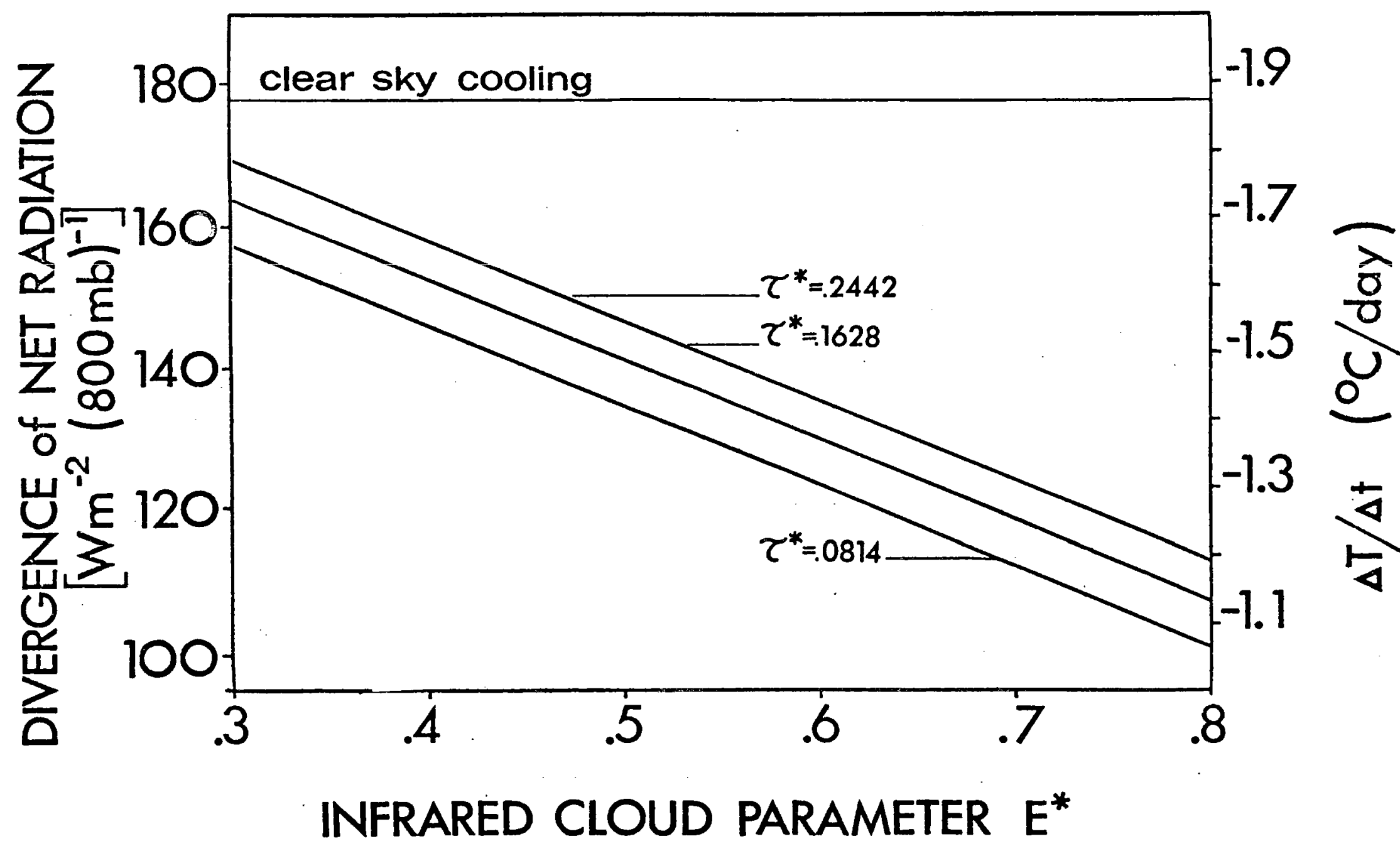

Figure 11. - Divergence of net radiation in the surface to cloud base layer as a function of cirrus radiative properties for $H=2 \mathrm{~km}, P_{B}=200 \mathrm{mb}$, lat. $15^{\circ} \mathrm{N}$, day 183 . 


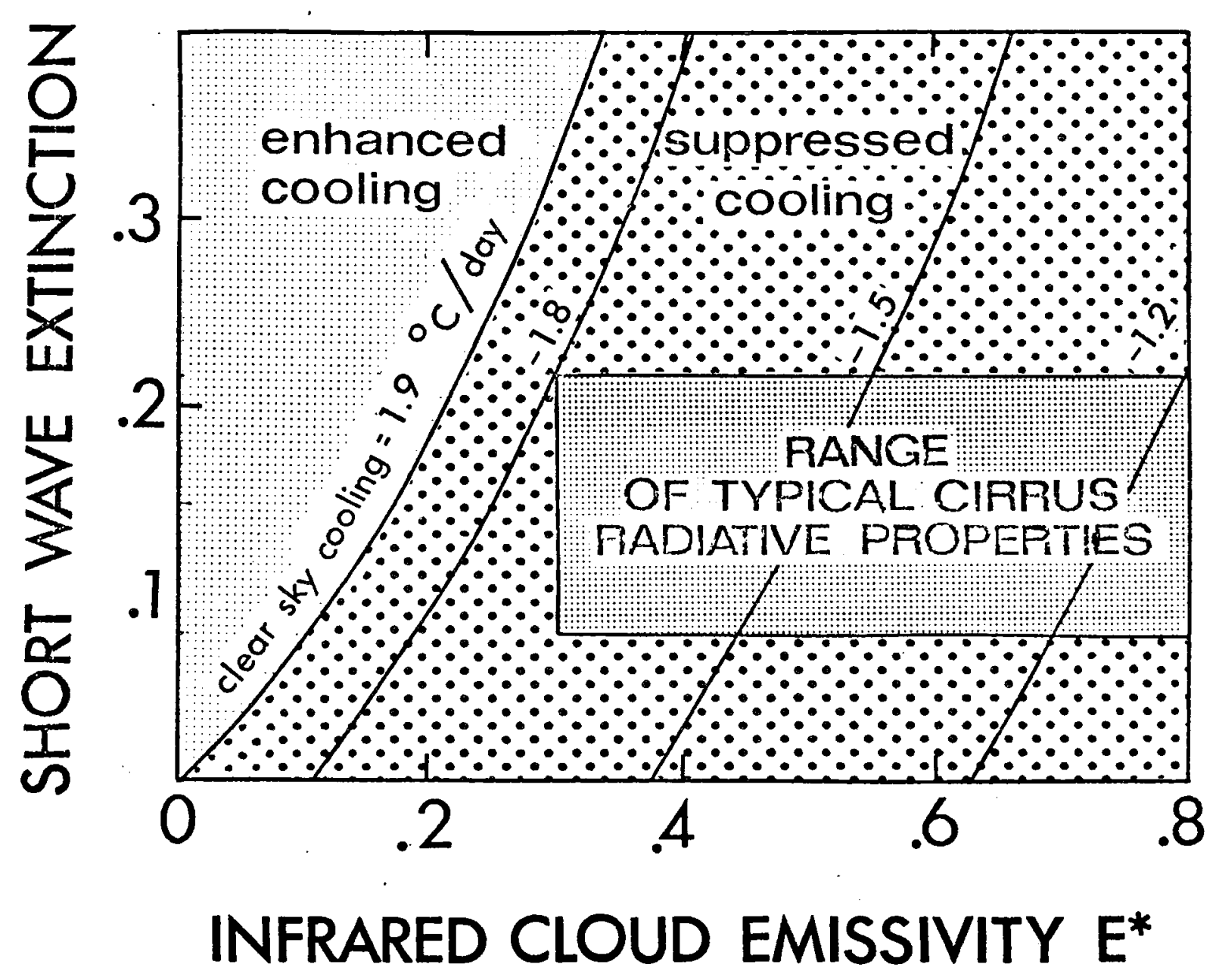

Figure 12. - Reinterpretation of Figure 11 to show those combinations of cirrus radiative properties that will give the equivalent clear sky cooling in the subcloud layer. 


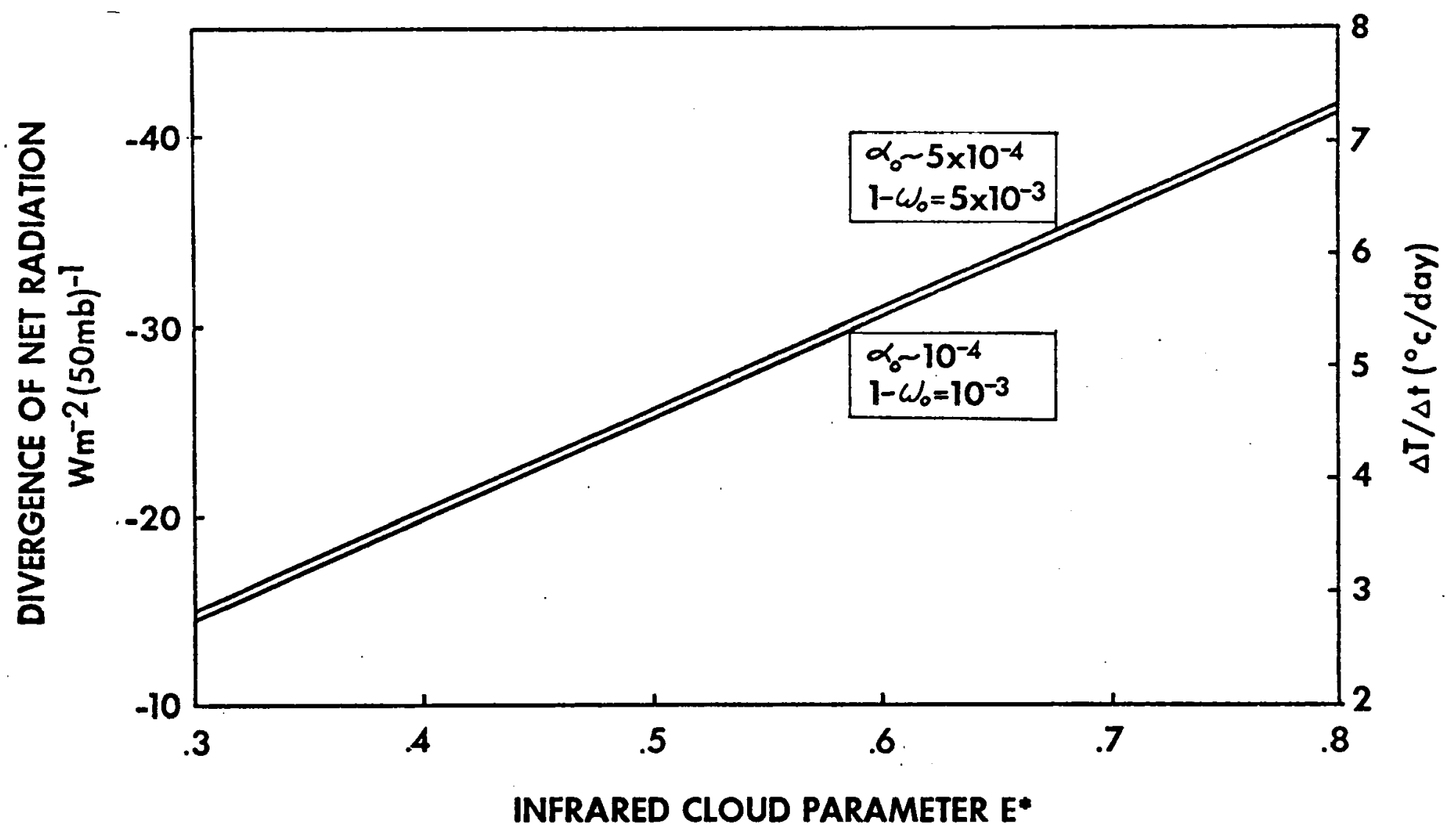

Figure 13. - Divergence of net radiation in the cloud layer itself as a function of cirrus radiative properties for

$H=2 \mathrm{~km}, P_{B}=200 \mathrm{mb}$, 1at. $15^{\circ} \mathrm{N}$, day 183. 


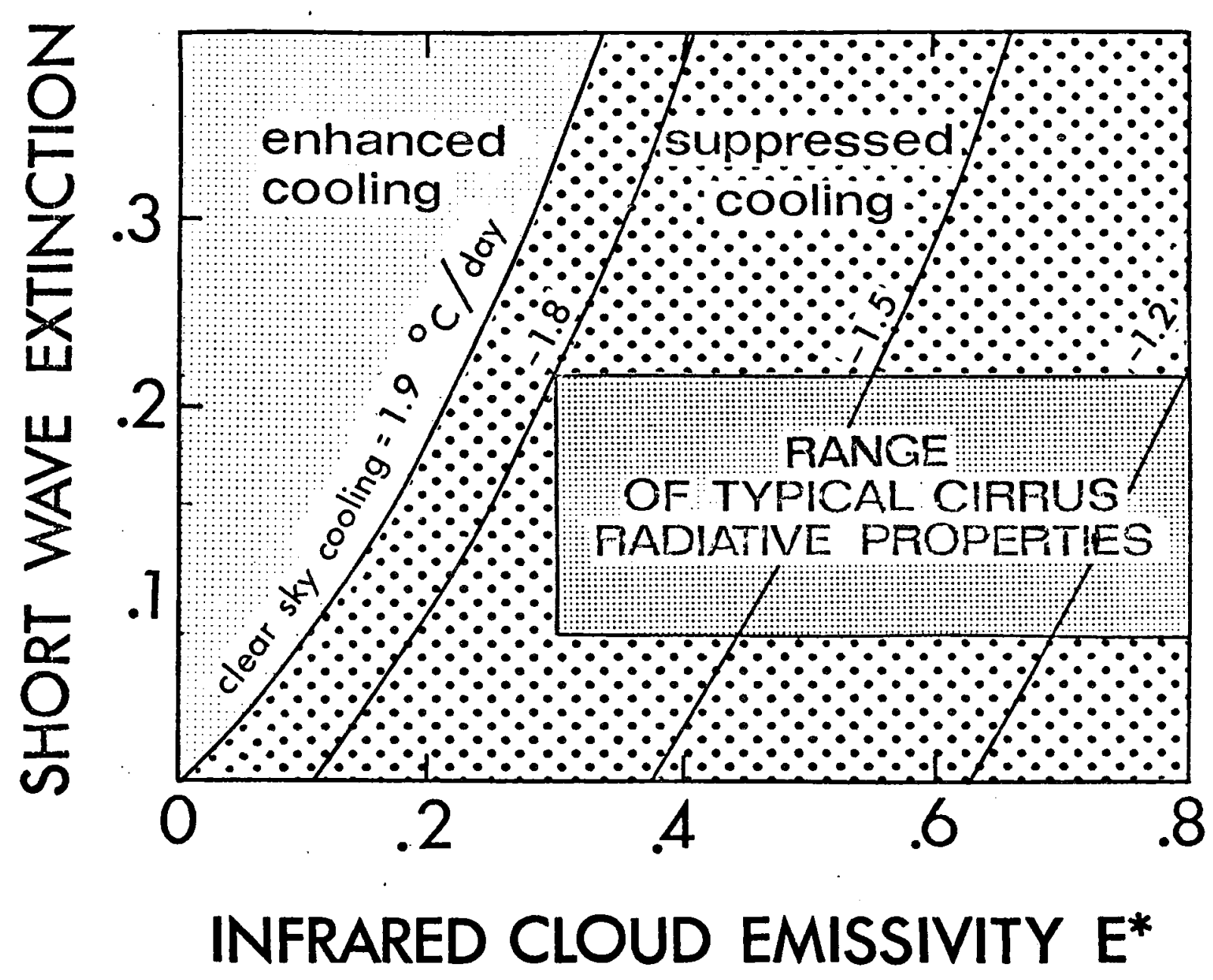

Figure 12. - Reinterpretation of Figure 11 to show those combinations of cirrus radiative properties that will give the equivalent clear sky cooling in the subcloud layer. 


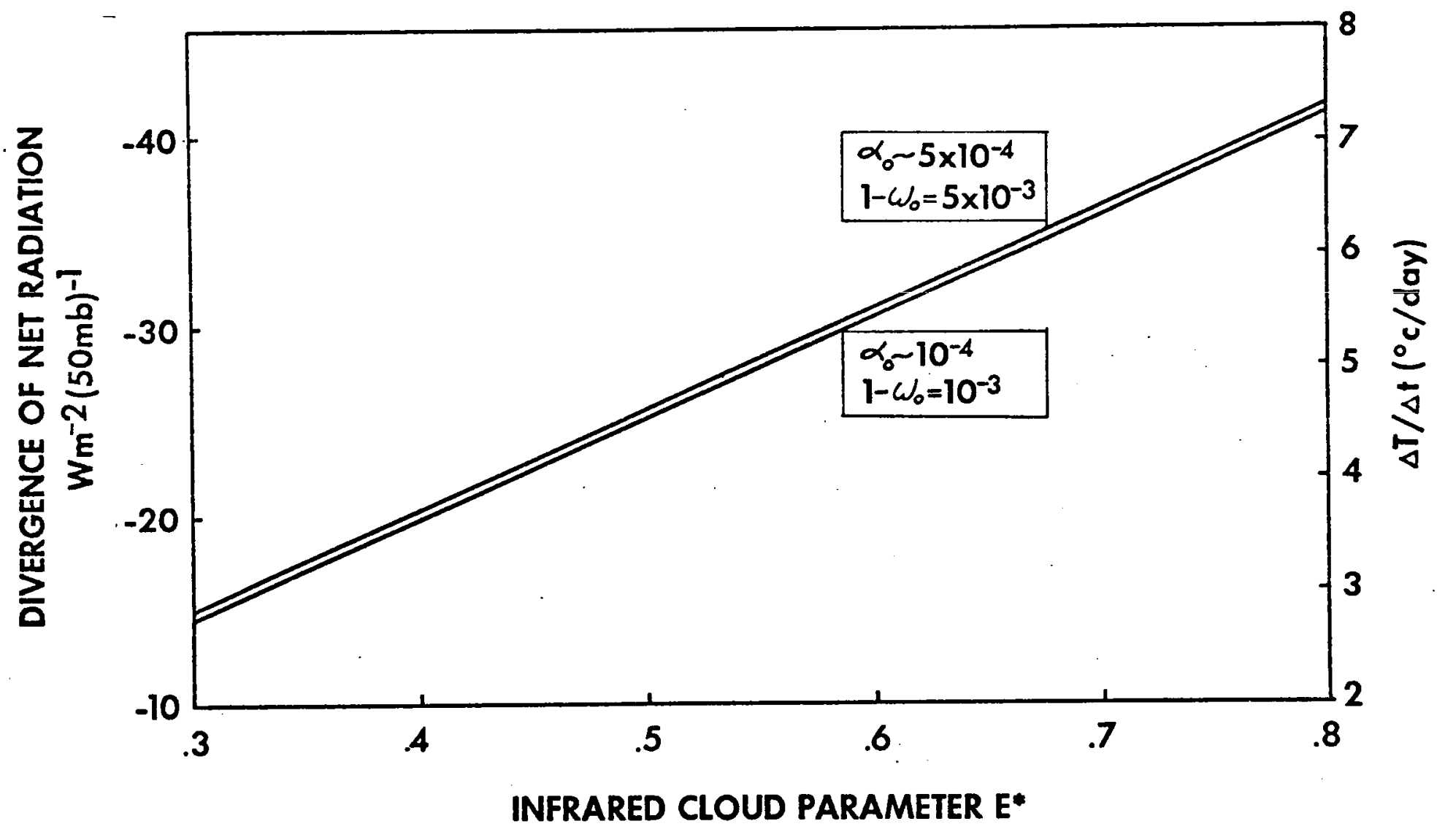

Figure 13. - Divergence of net radiation in the cloud layer itself as a function of cirrus radiative properties for $H=2 \mathrm{~km}, P_{B}=200 \mathrm{mb}$, 1at. $15^{\circ} \mathrm{N}$, day 183 . 


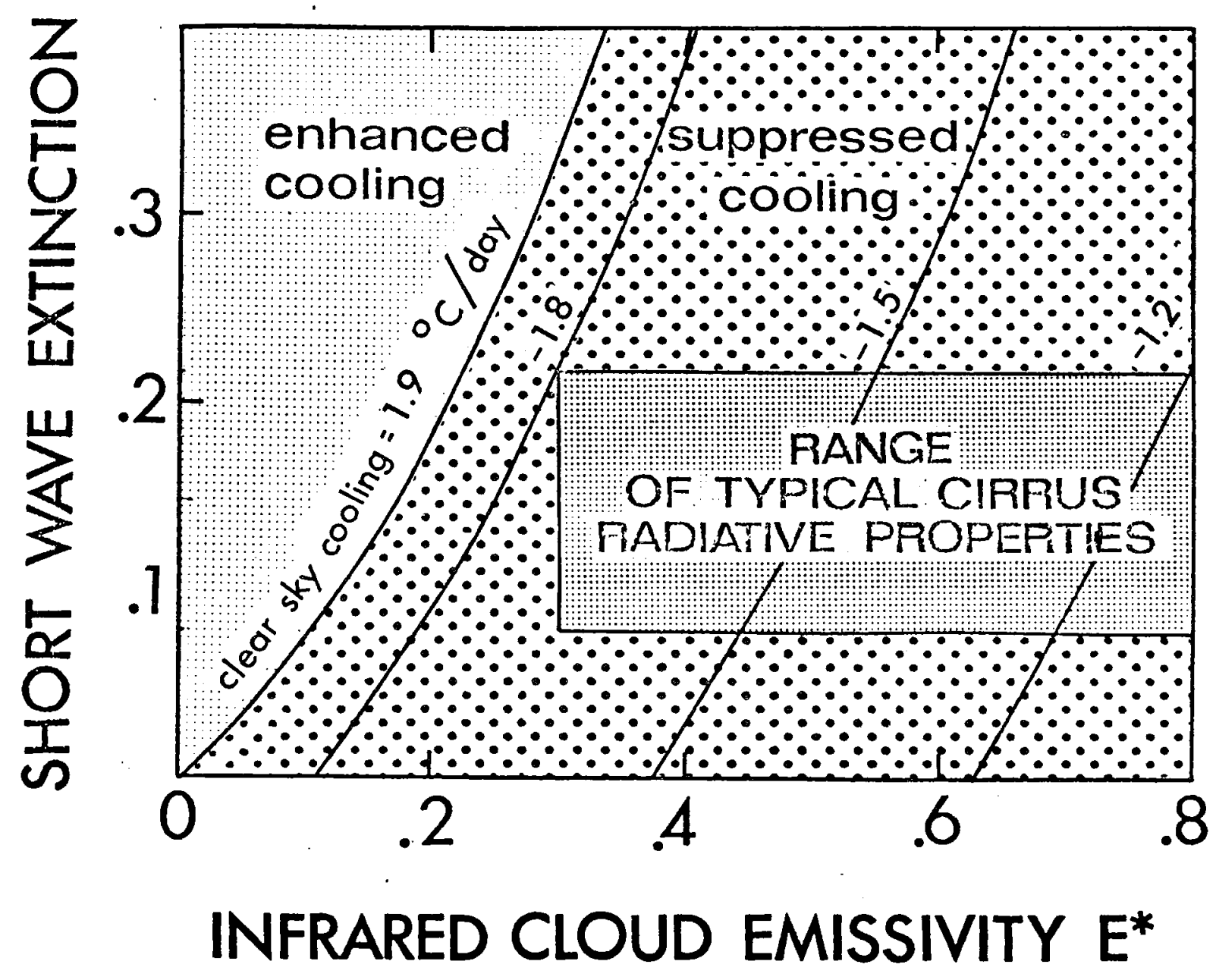

Figure 12. - Reinterpretation of Figure 11 to show those combinations of cirrus radiative properties that will give the equivalent clear sky cooling in the subcloud layer. 


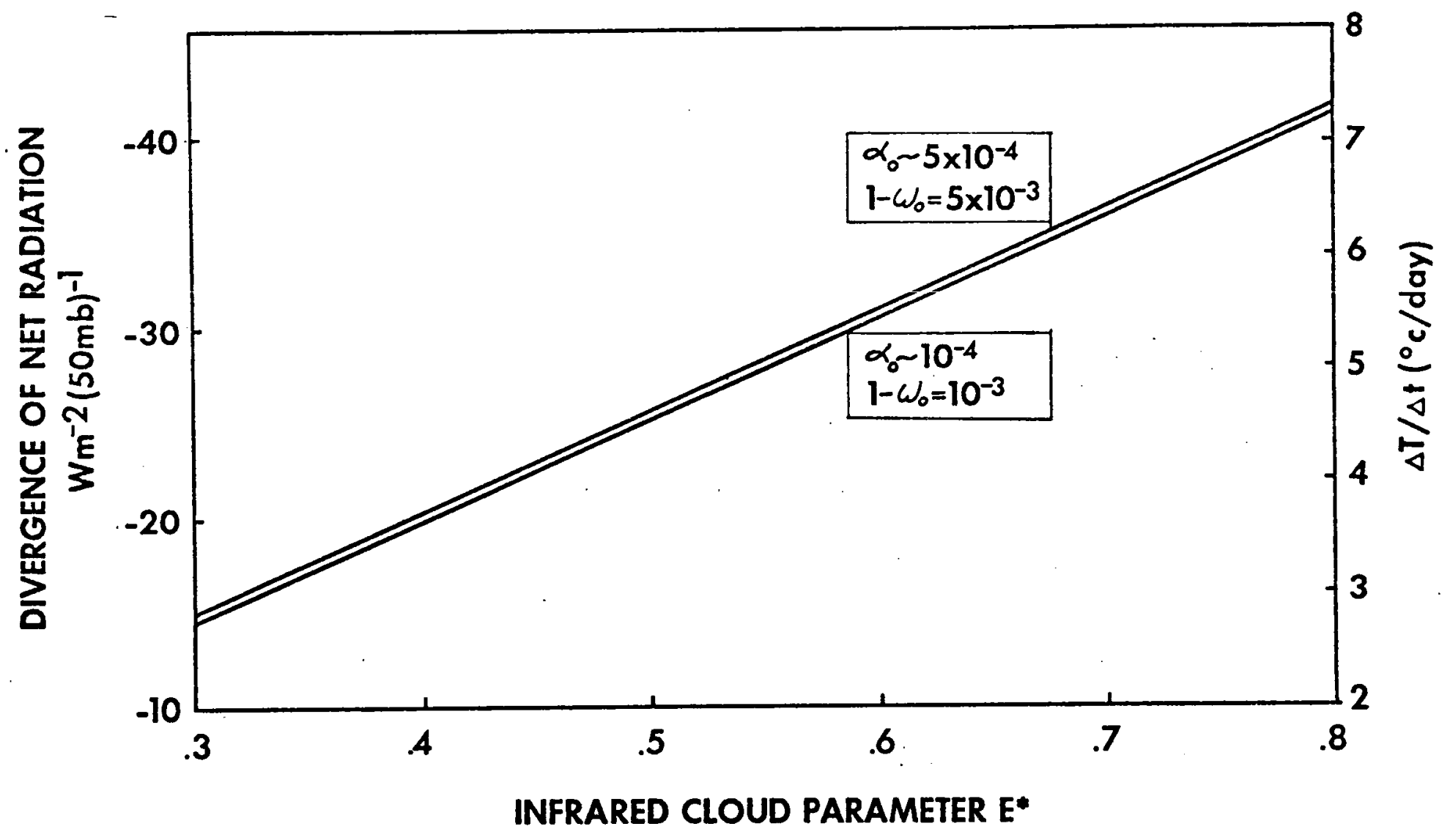

Figure 13. - Divergence of net radiation in the cloud layer itself as a function of cirrus radiative properties for

$\mathrm{H}=2 \mathrm{~km}, \mathrm{P}_{\mathrm{B}}=200 \mathrm{mb}$, 1at. $15^{\circ} \mathrm{N}$, day 183 . 


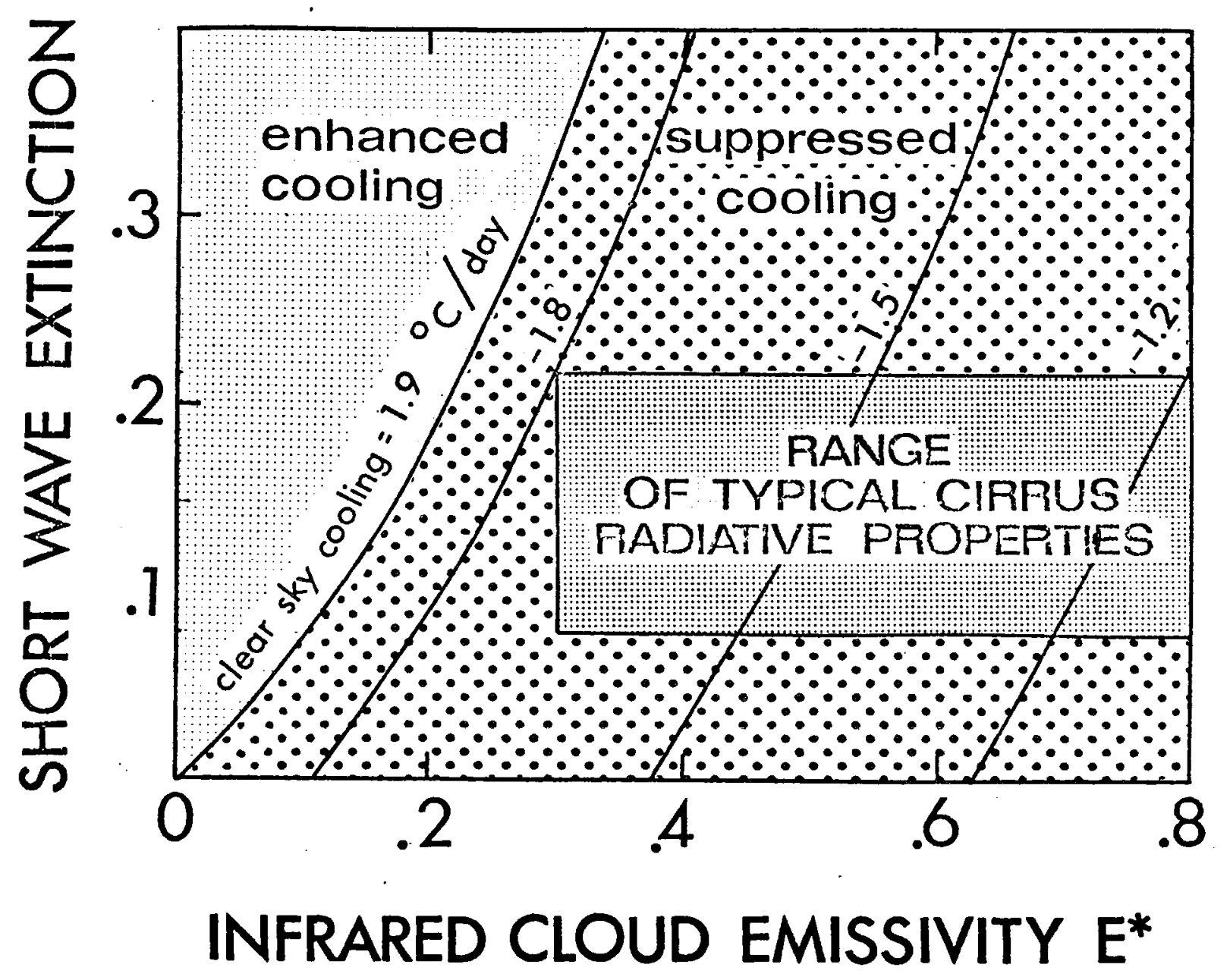

Figure 12. - Reinterpretation of Figure 11 to show those combinations of cirrus radiative properties that will give the equivalent clear sky cooling in the subcloud layer. 


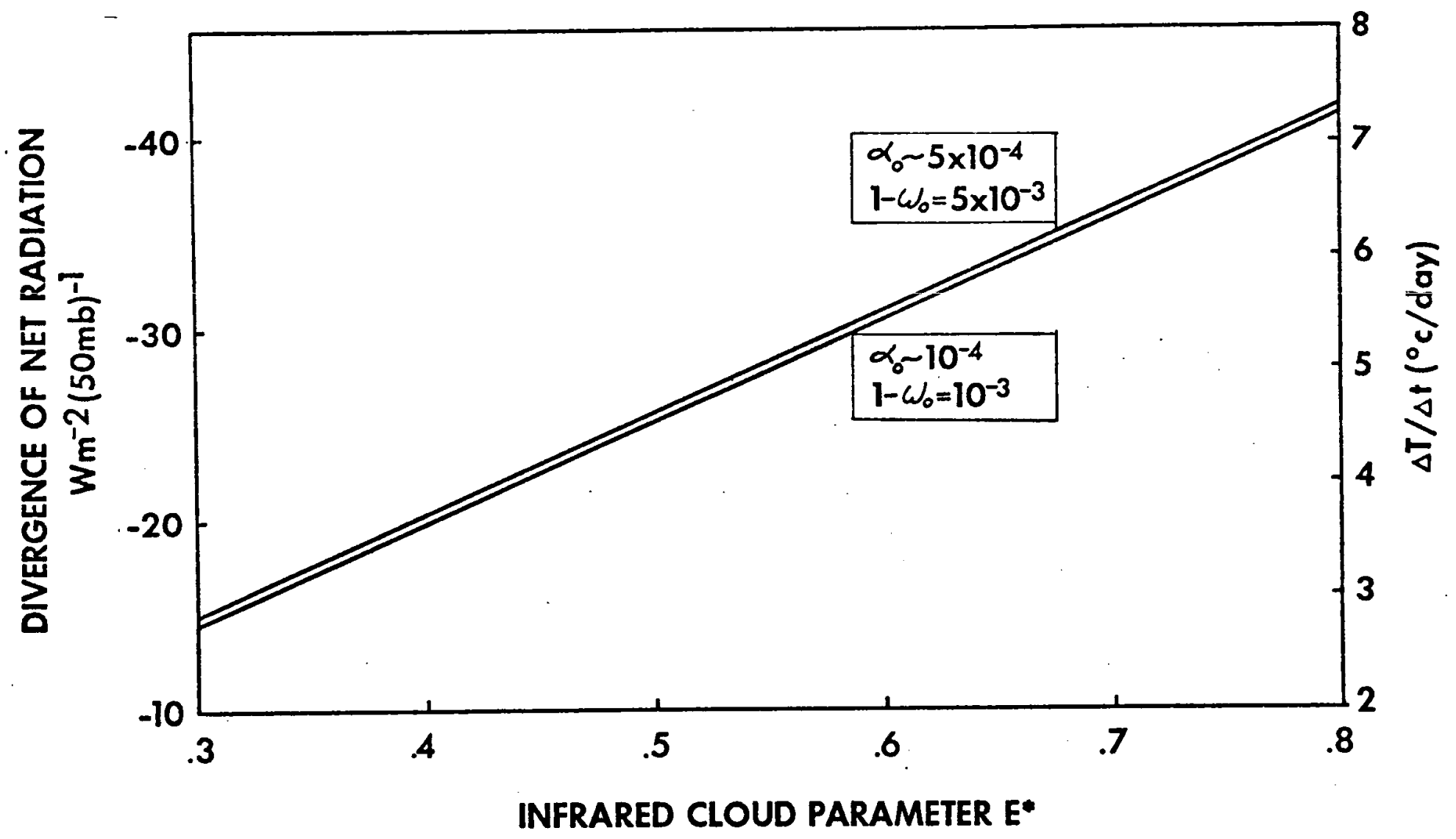

Figure 13. - Divergence of net radiation in the cloud layer itself as a function of cirrus radiative properties for $H=2 \mathrm{~km}, P_{B}=200 \mathrm{mb}$, lat. $15^{\circ} \mathrm{N}$, day 183 . 
where $\left(\frac{\Delta T}{\Delta t}\right)_{B L A C K}^{L W}$ is the infrared radiative temperature change of a cloud layer whose emissivity is unity. The dependence of the divergence on the absorptivity of the cirrus crystals is illustrated by the small increase in layer warming when $1-\omega_{0}$ is increased by a factor of five.

A $2 \mathrm{~km}$ thick cirrus cloud with $\tau^{*}=0.1628$ contains four grams of ice in a $1 \mathrm{~m}^{2}$ vertical column through the cloud. Allowing this ice to sublimate with a linear decrease of both cloud mass and radiative heating with time yields a total sublimation of the cloud ice mass in 48 minutes. The radiatively generated energy obviously must be dissipated to the air around each crystal if the cloud is to retain its structure over a long time period.

Possible explanations for the export of this radiatively generated energy from the cloud layer come from an examination of the first law of thermodynamics. With the local time derivatives of $T$ and $P$ zero and ignoring the horizontal pressure gradient, this is written

$$
\frac{\Delta T}{\Delta t}=\bar{u} \frac{\partial T}{\partial x}+(\gamma d-\gamma) \bar{w}+S
$$

where $\frac{\Delta T}{\Delta t}$ is the radiatively generated energy, $\bar{u}$ is the horizontal velocity of the air relative to the cirrus cloud particles, $\bar{w}$ is the vertical motion of the air through the cloud and $S$ represents other diabatic processes not explicitly considered. Horizontal motion of the air relative to the cloud particles and expansion of the air undergoing vertical motion through the cloud are assumed to account equally for the total export of energy generated radiatively. 
Considering $\bar{u}$ to be an eddy component of the horizontal wind having a velocity relative to the crystals of the cirrus cloud and allowing the air traversing $10 \mathrm{~km}$ of cirrus cloud to warm $1^{\circ} \mathrm{C}$ (within measurement accuracy) gives

$$
\bar{u}=\frac{1}{2} \frac{\Delta T / \Delta t}{\partial T / \partial x}=\frac{(.5)(2.8)}{(86400)\left(10^{-4}\right)} \mathrm{m} \mathrm{sec}^{-1}=0.2 \mathrm{~m} \mathrm{sec}^{-1} .
$$

This value is within measurement accuracy for drift velocities of clouds relative to the wind.

The vertical velocity necessary to dissipate $\frac{1}{2}$ of the radiative energy by expansion may be computed from

$$
\bar{w}=.5 \frac{1}{\gamma d-\gamma}\left(\frac{\Delta T}{\Delta t}\right)=\frac{(.5)(2.8)\left(10^{5}\right)}{(4)(86400)}=0.41 \mathrm{~cm} \mathrm{sec}^{-1}
$$

where $\gamma d$ is the adiabatic lapse rate of temperature $=10^{\circ} \mathrm{c} \mathrm{km}^{-1}$ and $\gamma$ is the actual lapse rate through the cloud $=6^{0} \mathrm{c} \mathrm{km}^{-1}$. $\bar{w}=0.3 \mathrm{~cm} \mathrm{sec}^{-1}$ is reasonable for the tropical atmosphere.

The divergence of radiation across cirrus layers of different geometric thicknesses and base pressures is illustrated in Figure 14 for $1-\omega_{0}=10^{-3}$ and for a range of emissivity indicated by the solid line in each case. The divergence in all cases tends toward zero as $\varepsilon^{*}$ goes to zero. A cloud of the same geometric thickness exhibits a greater negative divergence of net radiation at higher altitudes; thicker clouds with the same base pressure show a similar effect. This is caused by the decrease of the emitted $\varepsilon^{*} \sigma T_{T}{ }^{4}$ term at lower temperatures and its effect on the divergence of upward irradiance (c.f. Figure 10). Lower, warmer cirrus give radiative cooling at all values of $\varepsilon^{*}$ while the higher clouds warm radiatively. 


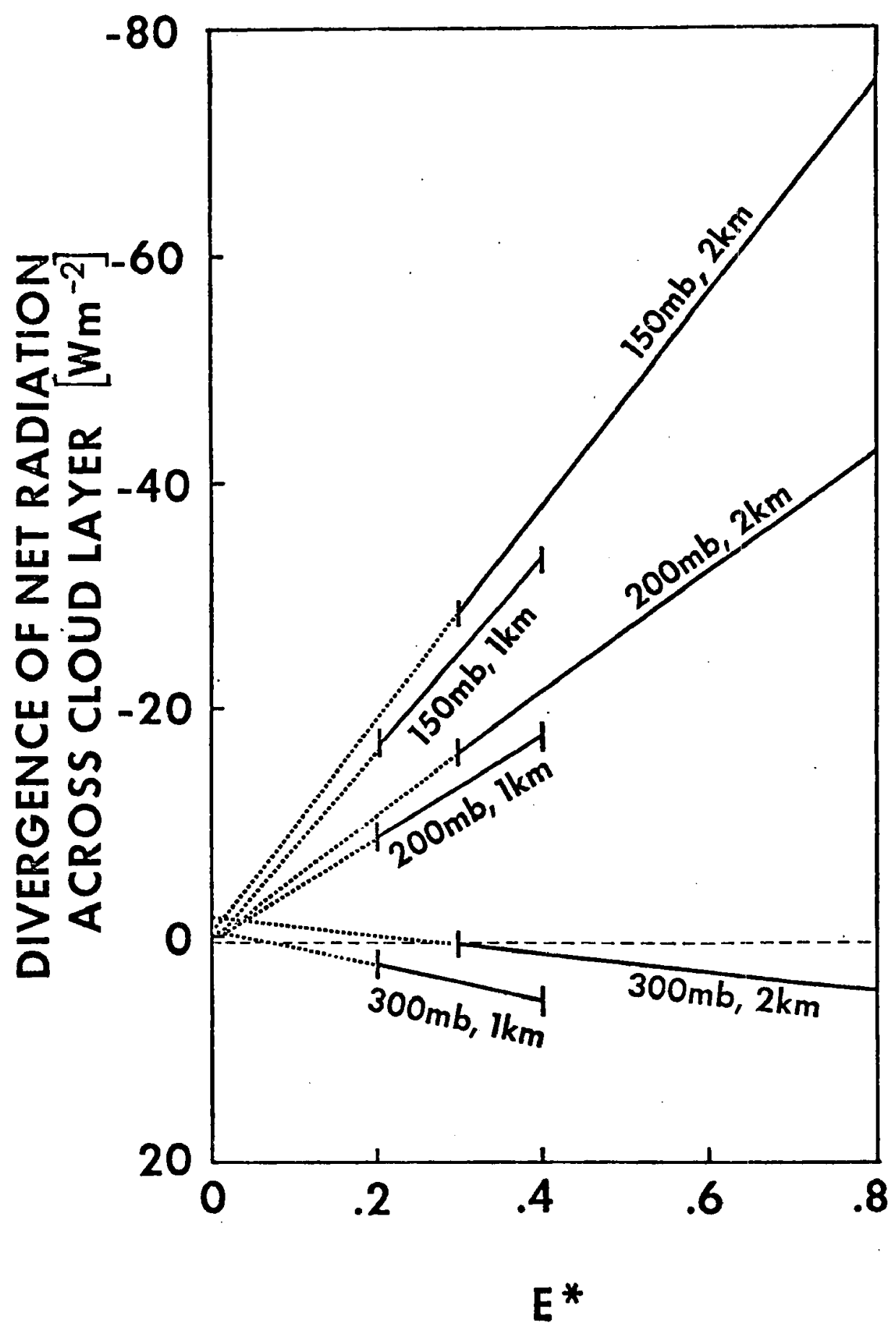

Figure 14. - The divergence of net radiation across cirrus clouds of different geometric thickness and base pressures for realistic ranges of $\varepsilon^{*}$ with $1-\omega_{0}=10^{-3}$. 
Above the cloud, in the layer from cloud top to the top of the atmosphere, the divergence is suppressed relative to the clear sky value. Figure 15 illustrates the linear decrease of divergence with decreasing emissivity. With $\varepsilon^{*}=.8$, the dominant $\varepsilon^{*} \sigma_{T}{ }_{T}^{4}$ term (see Figure 10) reduces the net irradiance at cloud top to a value less than the net irradiance at $10 \mathrm{mb}$, giving a longwave radiative temperature change of $-0.92^{\circ} \mathrm{C} \mathrm{day}^{-1}$. As $\varepsilon^{*}$ decreases, the difference in the net fluxes decreases as does the divergence. The shortwave heating in the layer increases with $\tau^{*}$. More energy is reflected by an optically thicker cloud thus increasing the amount absorbed in this above-cloud layer.

Figure 16 presents a comparison between the vertical profiles of daily averaged radiational temperature change for a cloudless tropical atmosphere and for the same atmosphere with a $2 \mathrm{~km}$ thick cirrus layer with a base pressure of $200 \mathrm{mb}$. The shortwave optical thickness of the cloud is 0.1628 and the longwave emissivity is 0.3 . The value of $\varepsilon^{*}$ was chosen to give the minimum radiative effect. The optical thickness lies in the center of the expected range of values. With cirrus clouds present, each layer of the atmosphere with the exception of the $50 \mathrm{mb}$ layer immediately above the cloud, warms radiatively more (or cools less) than that calculated for a cloudless atmosphere. The strong warming effect in the cloud layer itself (200-150 mb) is linearly dependent on cloud infrared emissivity as shown in equation (22).

A numerical problem in the computation of irradiance by the model for the layers of the atmosphere immediately adjacent to the cloud was discovered and the transfer of longwave radiation through 


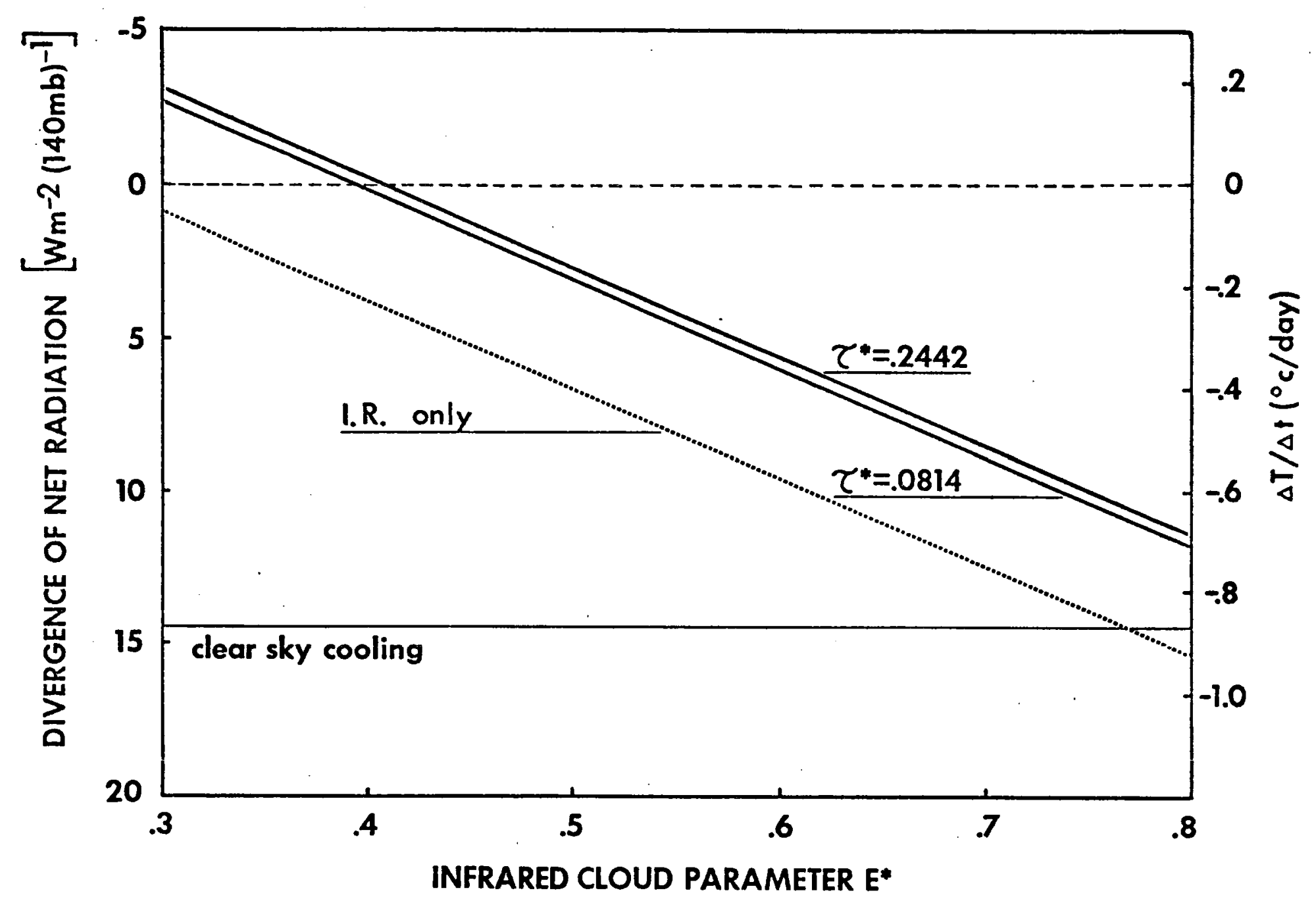

Figure 15. - Divergence of net radiation above the cloud layer as a function of cirrus radiative properties. 


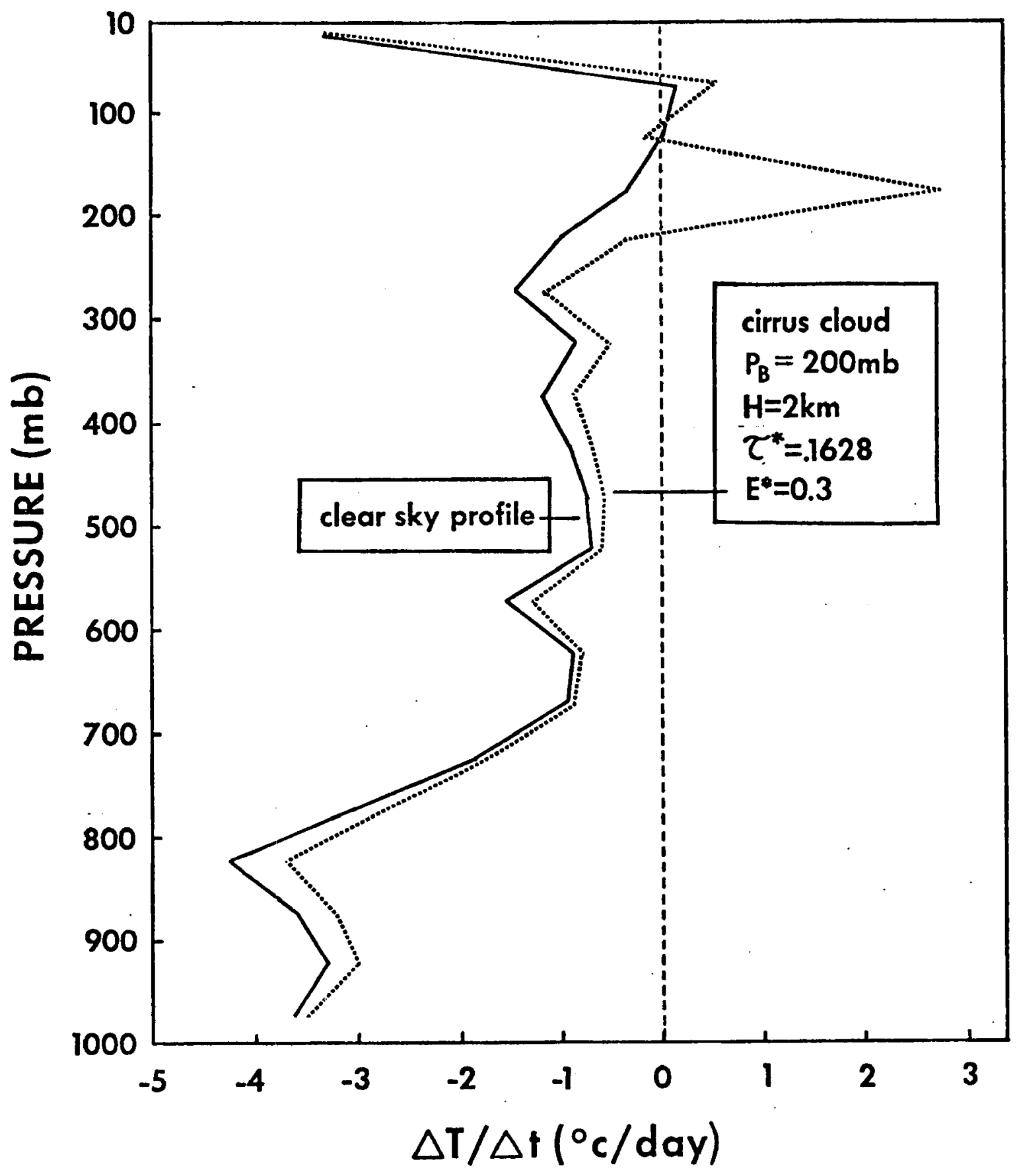

Figure 16. - Vertical profiles of daily averaged radiational temperature change for a cloudless tropical atmosphere and for the same atmosphere with cirrus. 
these layers was calculated using spectral emissivities. Because the assumption of grey body longwave irradiance at the cirrus cloud boundary is not entirely realistic for the $550-800 \mathrm{~cm}^{-1}$ absorption band of carbon dioxide, the model underestimates the irradiance at cloud base in this spectral interval. Emission by $\mathrm{CO}_{2}$ gas at warmer temperatures in the layer below the cloud gives an anomalous increase in the downward irradiance at $250 \mathrm{mb}$. The divergence of this radiation gives a physically unrealistic value of cooling for this first subcloud layer. Similarly, the transmission of upward irradiance through the cloud and the grey body partitioning of this radiation gives an anomalous increase of irradiance in the $\mathrm{CO}_{2}$ band. Absorption and re-emission by carbon dioxide at colder environmental temperatures above the cloud give a physically unrealistic convergence of radiation in this first layer.

To correct this shortcoming, the downward irradiance in the first layer below the cloud and the upward irradiance in the first layer above the cloud in the carbon dioxide band from $550-800 \mathrm{~cm}^{-1}$ were investigated from the initial conditions at the cloud boundaries using a longwave transfer model with $10 \mathrm{~cm}^{-1}$ spectral resolution. For example:

$$
\begin{aligned}
& { }^{H \uparrow}{ }_{100}\left(\mathrm{CO}_{2}\right)=\sigma \mathrm{T}_{1}{ }^{4} \Delta \varepsilon_{1}+
\end{aligned}
$$

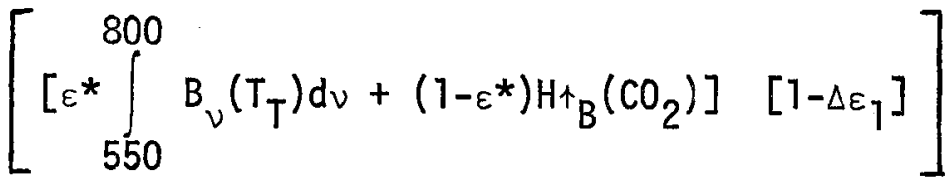

where $\Delta \varepsilon_{1}$ is the emissivity of $\mathrm{CO}_{2}$ in the first layer of the atmosphere above the cloud. The first term represents emission by carbon dioxide in the first $50 \mathrm{mb}$ layer of the atmosphere above 
the cloud; the second term is the irradiance at cloud top in the $\mathrm{CO}_{2}$ band which is transmitted through this layer. The $550-800 \mathrm{~cm}^{-1}$ carbon dioxide band shows a relative divergence of upward irradiance above the cloud. This divergence (cooling) increases with increasing $\varepsilon^{*}$. The downward irradiance below the cloud in this spectral region exhibits a negative divergence (warming) that increases with $\varepsilon^{\star}$.

Equations similar to (26) were developed for the spectrally integrated longwave irradiance at the boundaries of these two layers. Equation (21) was then applied to yield the longwave radiative temperature change. For a black cloud with the same geometric characteristics as in Figure $16,\left(\frac{\Delta T}{\Delta t}\right)_{L W}=+0.88^{\circ} \mathrm{C} \mathrm{day}^{-1}$ in the first layer below the cloud and $\left(\frac{\Delta T}{\Delta t}\right)_{L W}=-0.67^{\circ} \mathrm{C} \mathrm{day}^{-1}$ in the layer immediately above the cloud. Shortwave heating is increased above a cirrus cloud due to absorption of the reflected component. This effect moderates the longwave cooling. Because the longwave irradiance quickly regains its spectral characteristics due to emission by gaseous components of the atmosphere, the cooling rate computations for other layers of the atmosphere further removed from the cloud layer are expected to be physically realistic.

\section{B. Parameterization of Cirrus Effects on Radiative Divergence}

The radiative temperature change in three layers of the atmosphere for the cirrus clouds of Table 2 has been parameterized in terms of the thickness of the cloud layer and the temperature of the cloud base. The range of cloud radiative properties is determined by these physical inputs. The maximum and minimum radiative temperature change for the three layers previously 
defined is shown as a function of cloud base temperature for $1 \mathrm{~km}$ thick cirrus in Figure 17 and for cirrus clouds $2 \mathrm{~km}$ thick in Figure 18. The combinations of $\tau^{*}$ and $\varepsilon^{*}$ which give the maximum radiative temperature change for each of the three layers is shown in Table 3.

Table 3: Combination of $\tau^{*}$ and $\varepsilon^{*}$ which give the maximum radiative temperature change for each of the three layers of Figures 17 and 18.

\begin{tabular}{|c|c|c|}
\hline \multirow[b]{2}{*}{ ATMOSPHERIC LAYER } & \multicolumn{2}{|c|}{ MAXIMUM $\Delta T / \Delta t$} \\
\hline & $\tau^{*}$ & $\varepsilon^{\star}$ \\
\hline Below Cloud & Smallest & Largest \\
\hline Within Cloud & Smallest & Largest \\
\hline Above Cloud & Largest & Snallest \\
\hline
\end{tabular}

(1) Smaller cloud optical thickness will attenuate less shortwave energy allowing it to penetrate through the cloud and be absorbed in the subcloud layer. Larger values of $\varepsilon^{*}$ increase the downward irradiance at cloud base while decreasing the loss of infrared radiation from the warmer underlying layers. The result is a maximum of radiative temperature change.

(2) Smaller $\tau^{*}$ gives less shortwave reflection at the cloud top. This allows more energy to be absorbed by the molecular constituents within the cloud. The increase with $\varepsilon^{*}$ of radiative warming in high cold cirrus cloud layers is due mainly to the reduction of the transmitted component of the upward irradiance at cloud top when $\varepsilon^{*}$ is large and the resulting convergence of net radiation. (3) In the above cloud layer, the larger $\tau^{*}$ reflects more energy 


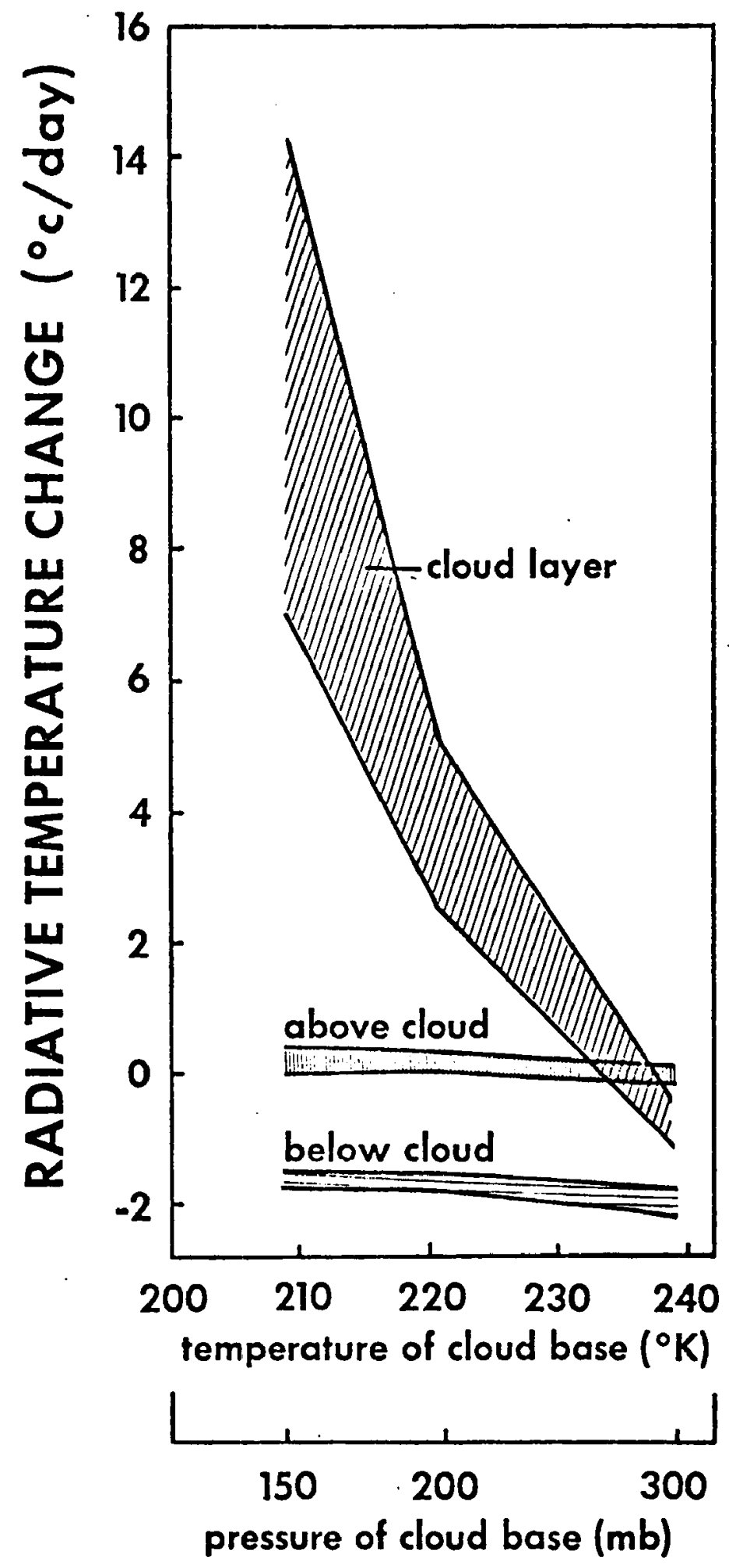

\section{$1 \mathrm{~km}$ thick cirrus}




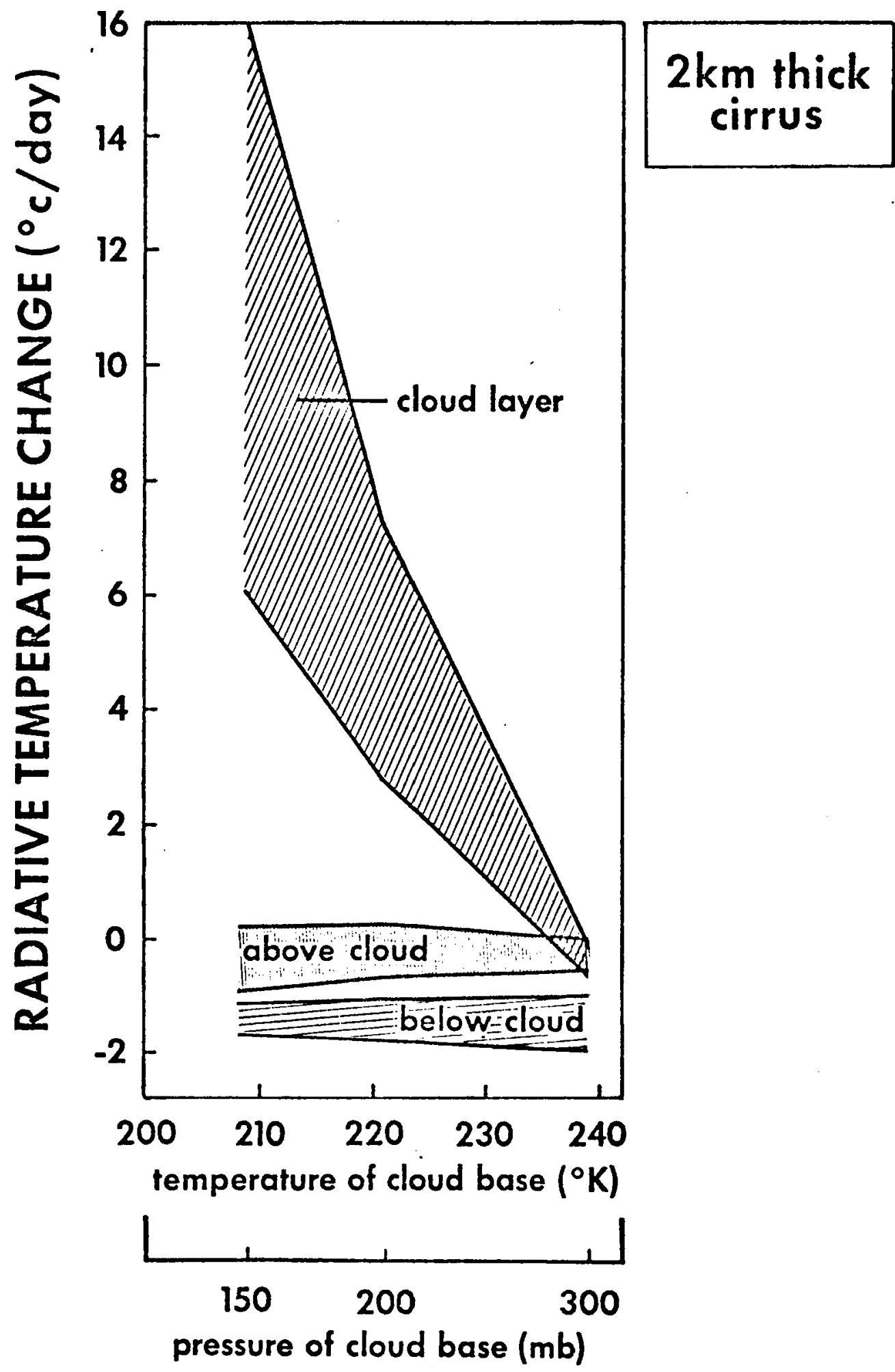

Figure 18. - Maximum and minimum radiative temperature change for three layers of the atmosphere as a function of cloud base location for $2 \mathrm{~km}$. thick cirrus. 
back into the layer allowing more shortwave absorption to occur. The smaller values of $\varepsilon^{*}$ give a greater transmission of below cloud radiation through to the atmosphere above the cloud and a greater relative convergence of longwave radiation in this layer.

\section{Radiative Energy Budget of the Tropical Atmosphere}

The total radiative energy budget at the surface of the sea and at the top of the atmosphere is determined for the cloudless tropical atmosphere and the same atmosphere with cirrus clouds present. Net shortwave and longwave radiative energy budgets are constructed at the top and bottom of the atmosphere to yield the divergence of total radiation in the atmosphere in one day as a function of cirrus cloud radiative properties. July 2 , the midpoint of the calendar year, was chosen for computational purposes.

The net flux of shortwave energy at the sea surface is determined at each zenith angle by the downward irradiance multiplied by $[1-A(\theta)]$. The albedo $A(\theta)$, employed is given in Appendix B. Computations for the clear sky and for three optical thicknesses of cirrus clouds are shown in Figure 19. The net irradiance is strongly dependent on the optical thickness of the cloud layer because the transmissivity of the cirrus cloud is exponentially dependent on $\tau^{*}$ (equation (12)), and the transmissivity of the entire atmosphere is drastically reduced as the optical thickness of the cirrus cloud layer increases.

Curves similar to those in Figure 19 were computed for the net shortwave irradiance at the top of the atmosphere. Here the net irradiance is the difference between the downward radiation from space and the irradiance, reflected from the cirrus cloud and 


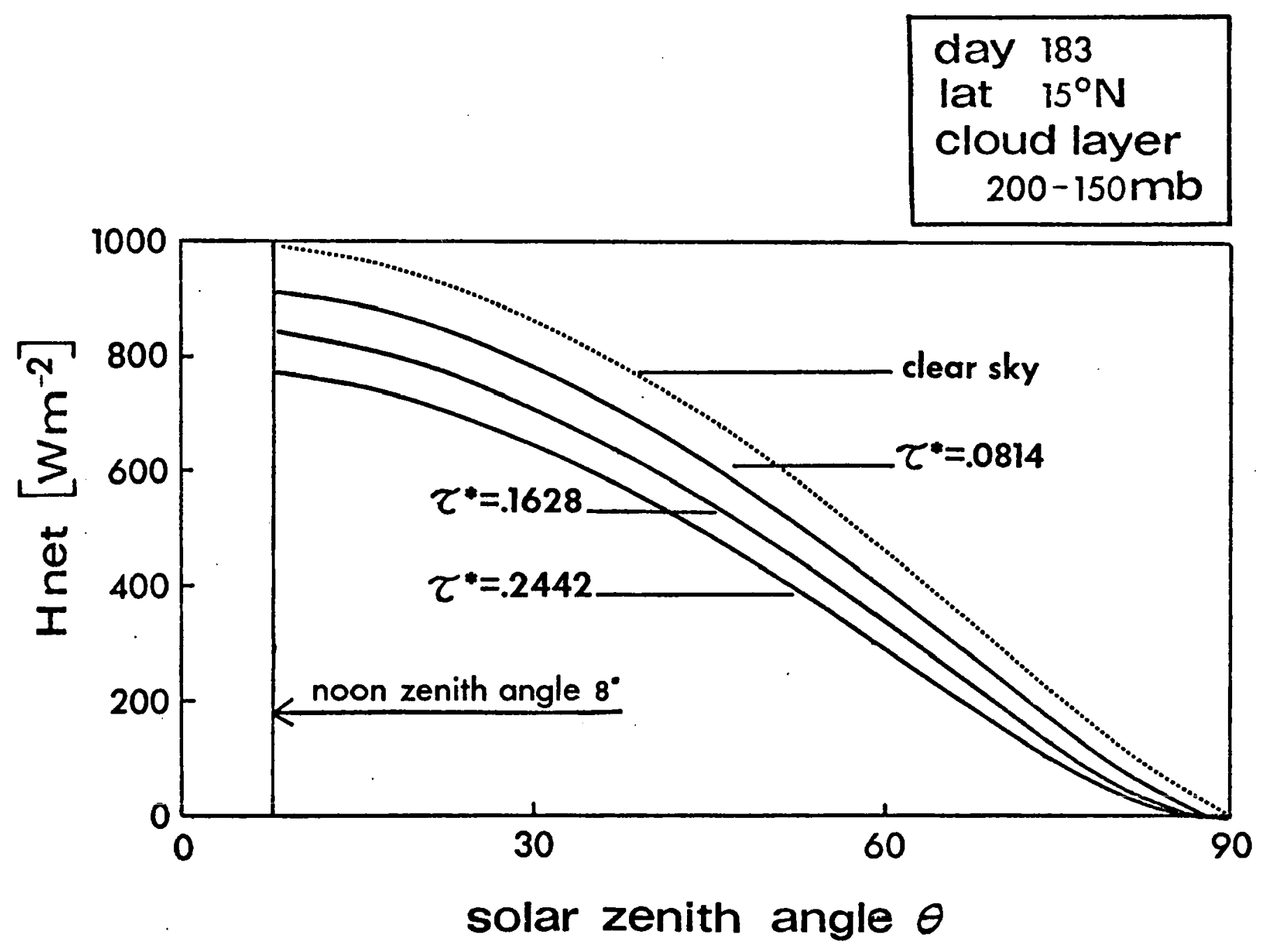

Figure 19. - Net shortwave irradiance at the sea surface vs. solar zenith angle for the cloud free atmosphere and for three different cirrus clouds. 
from the sea surface, which is transmitted through the atmosphere. Several factors allow the net irradiance at the top of the atmosphere to decrease slowly with increasing optical thickness. With cirrus clouds present, the increase of cloud reflectivity with solar zenith angle is balanced by the decrease of sea surface albedo and the reduced atmospheric transmissivity. As a result, ${ }^{H}$ SW TOP increases only slightly over the upward flux of shortwave irradiance in the clear atmosphere. In the cloudless atmosphere, ${ }^{H}{ }_{T O P}^{S W}$ is a result of Rayleigh scattering by molecules and large transmissivity of the atmosphere combined with a larger sea surface albedo for global radiation.

The net irradiance integrated over all zenith angles gives the net shortwave energy at the top and bottom of the atmosphere. Figure 20 shows net shortwave energy vs. $\tau^{*}$ for the cases described above. The more rapid decrease of net energy at the sea surface is apparent. The energy absorbed by the atmosphere is the difference of the two curves. This is illustrated in Figure 21 . The atmosphere with cirrus clouds present (solid black line) absorbs more shortwave energy than the cloud-free atmosphere.

The second component of the total energy budget is the net longwave radiation. The net longwave energy at the top of the atmosphere and at the sea surface is dependent on the cloud infrared' emissivity and the surface temperature. The atmosphere's temperature and moisture structure are held constant through the computations.

Because of the large amounts of water vapor present in the lower levels of the tropical atmosphere employed, the emissivity 


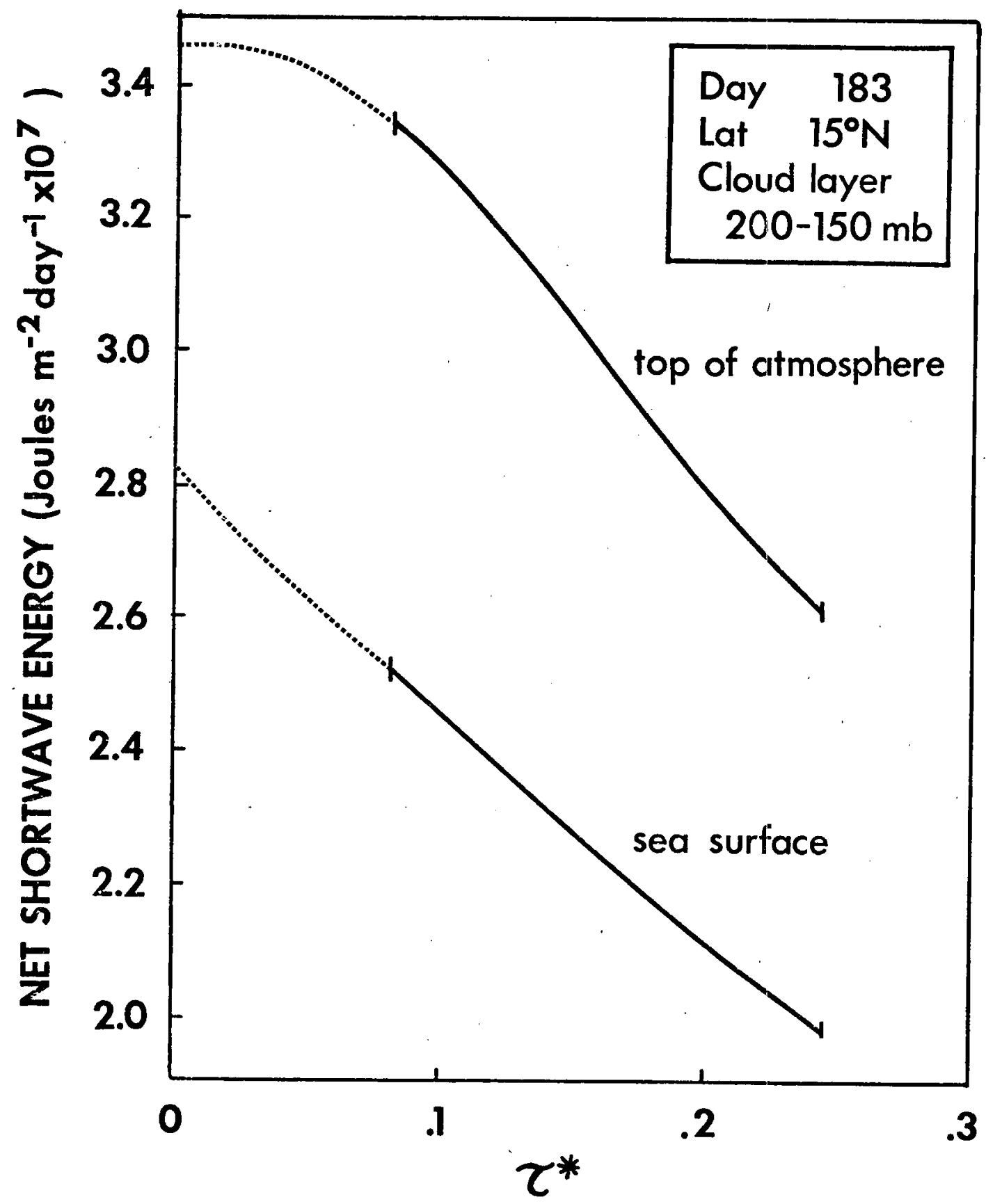

Figure 20. - Net shortwave energy at the sea surface and at the top of the atmosphere for different: cloud optical thicknesses. The solid lines refer to realistic cirrus clouds. 


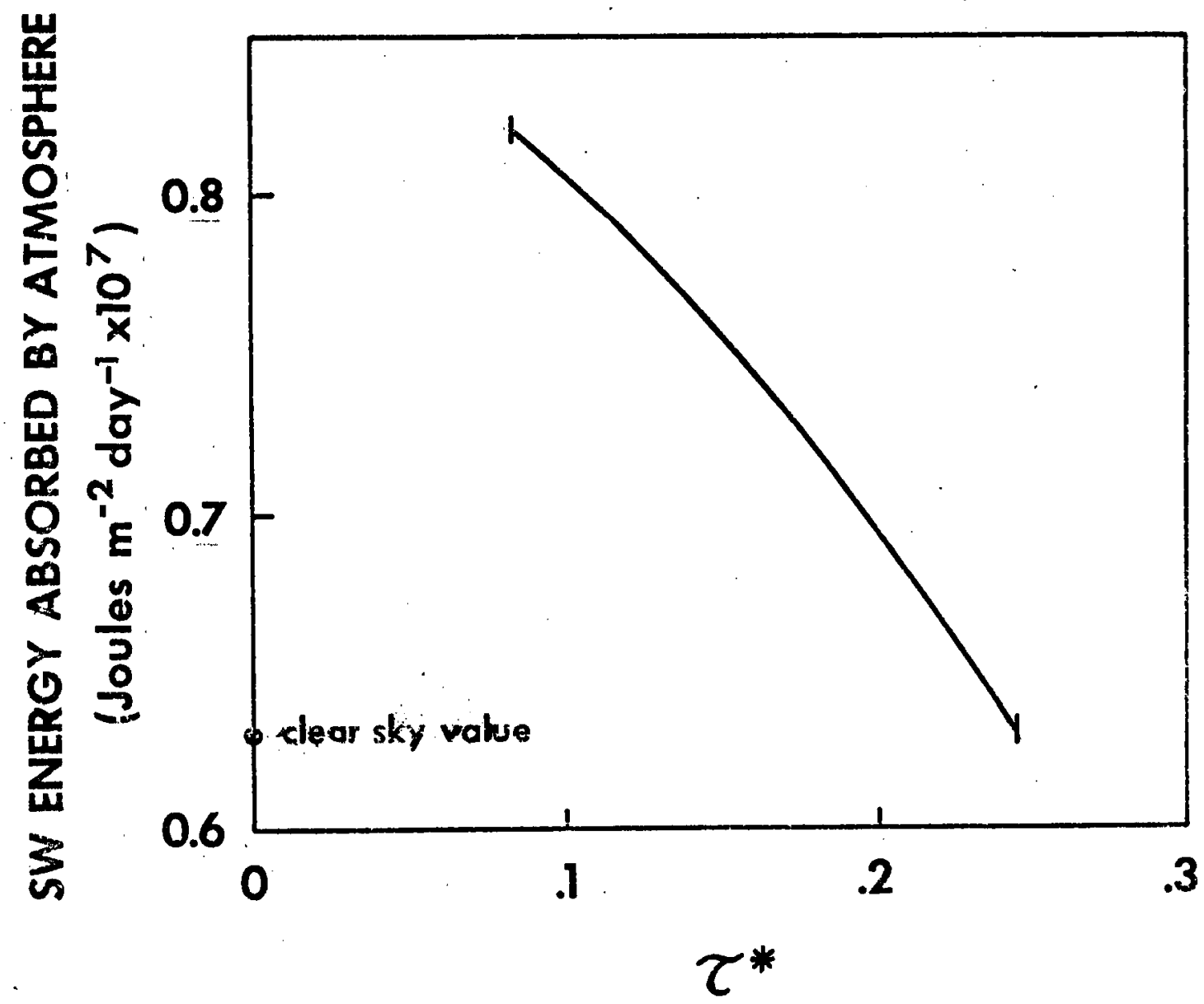

Figure 21. - Shortwave energy absorbed by the atmosphere for different cloud optical thicknesses; formed by differencing the curves of Figure 19. 
of the continuum (8-12 $\mu \mathrm{m})$ is large (Cox, 1973) and the downward, longwave irradiance at the surface is insensitive to the emissivity of a high cirrus cloud layer; increasing $0.2 \%$ from the clear to the $\varepsilon^{*}=0.8$ case. It is sensitive, however, to the temperature of the sea surface since the surface temperature directly influences the average temperature of the first layer of the atmosphere above the surface. The upward flux of longwave radiation at the surface depends on the emissivity of the surface and on its infrared reflectivity. The infrared emissivity used was assumed to be 0.97 and the infrared reflectivity of the sea surface 0.03 , as given by Anderson (1954). The net longwave flux at the surface is then given by

$$
H_{n_{S F C}}=H_{\downarrow}-\left[0.97 \sigma T_{s}^{4}+0.03 H_{\downarrow}\right]
$$

The longwave radiation at the top of the atmosphere is relatively independent of surface temperature because of the water vapor pressure broadened continuum but depends strongly on the $\varepsilon^{*}$ of the cirrus layer. Again we assume that the atmospheric temperature and moisture profiles remain constant.

The total radiation budget of the sea surface then is dependent primarily on the shortwave optical thickness of the cirrus cloud and on the sea surface temperature. Figure 22 shows this dependence for three values of the surface temperature. The variation of the net energy is seen to be equally dependent on the longwave and shortwave budgets.

At the top of the atmosphere the radiative energy budget is dependent primarily on the radiative properties and temperature of 


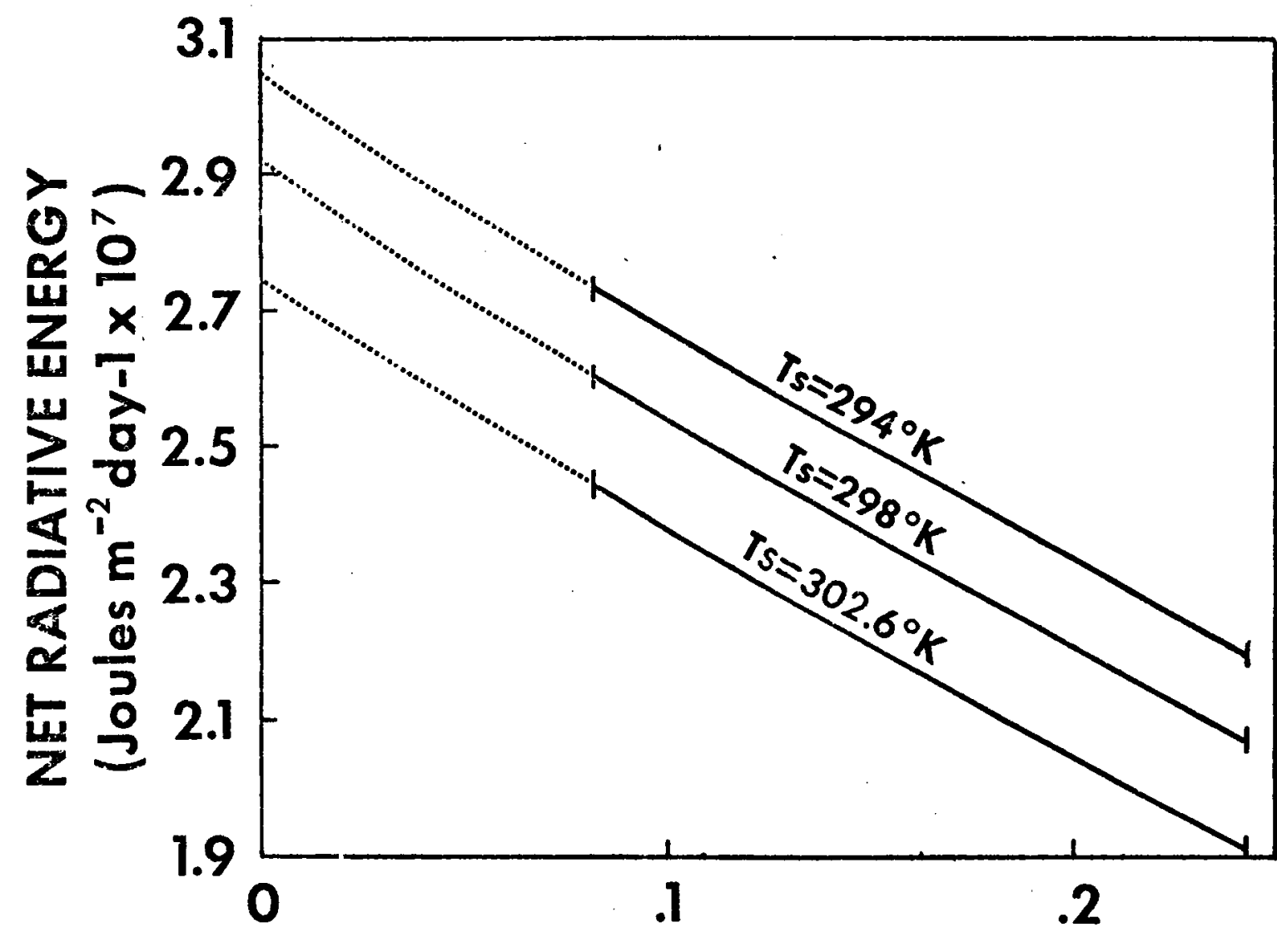

CLOUD OPTICAL THICKNESS $\tau^{*}$

Figure 22. - Net radiative energy at the sea surface as a function of $\tau^{*}$ for three values of the sea surface temperature. 
the cirrus cloud. An increase of ocean surface temperature of $8^{\circ} \mathrm{K}$ gives virtually no increase of upward flux at the cirrus cloud base. This dependence is illustrated in Figure 23 for realistic viaues of $\tau^{*}$ and $\varepsilon^{*}$. Again both the shortwave and longwave properties equally control the total radiative energy budget.

Figure 24a shows the shortwave and longwave radiative energy budgets at four levels of the atmosphere for the cloudless case and with a $2 \mathrm{~km}$ thick cirrus cloud $\left(\varepsilon^{*}=0.3, \tau^{*}=0.1628\right)$ present. The net shortwave energy above the cloud is reduced because of the cloud's reflectivity. Longwave losses are also reduced because of the low emission temperature of the cirrus cloud. The result is a slight reduction in the total net radiative energy at the top of the atmosphere from that expected in the cloudiess atmosphere. Increased cirrus emissivity will decrease the longwave losses at the top of the atmosphere while increased cloud optical thickness will increase the loss of shortwave radiation by reflection at the cloud layer. At the bottom of the atmosphere, the net radiative energy is decreased when cirrus clouds are present because of the reduced transmissivity of the atmosphere for shortwave radiation. Reflection by the cloud is the main factor reducing the energy incident at the surface. The longwave surface budget is insensitive to the presence of the cloud layer.

The net energy at four levels is presented in Figure 24b along with the net energy change and radiative temperature change for three layers encompassing the atmosphere. The convergence of radiative energy in the cirrus cloud layer is particularly clear. Below the cloud the divergence of total net radiative energy is 


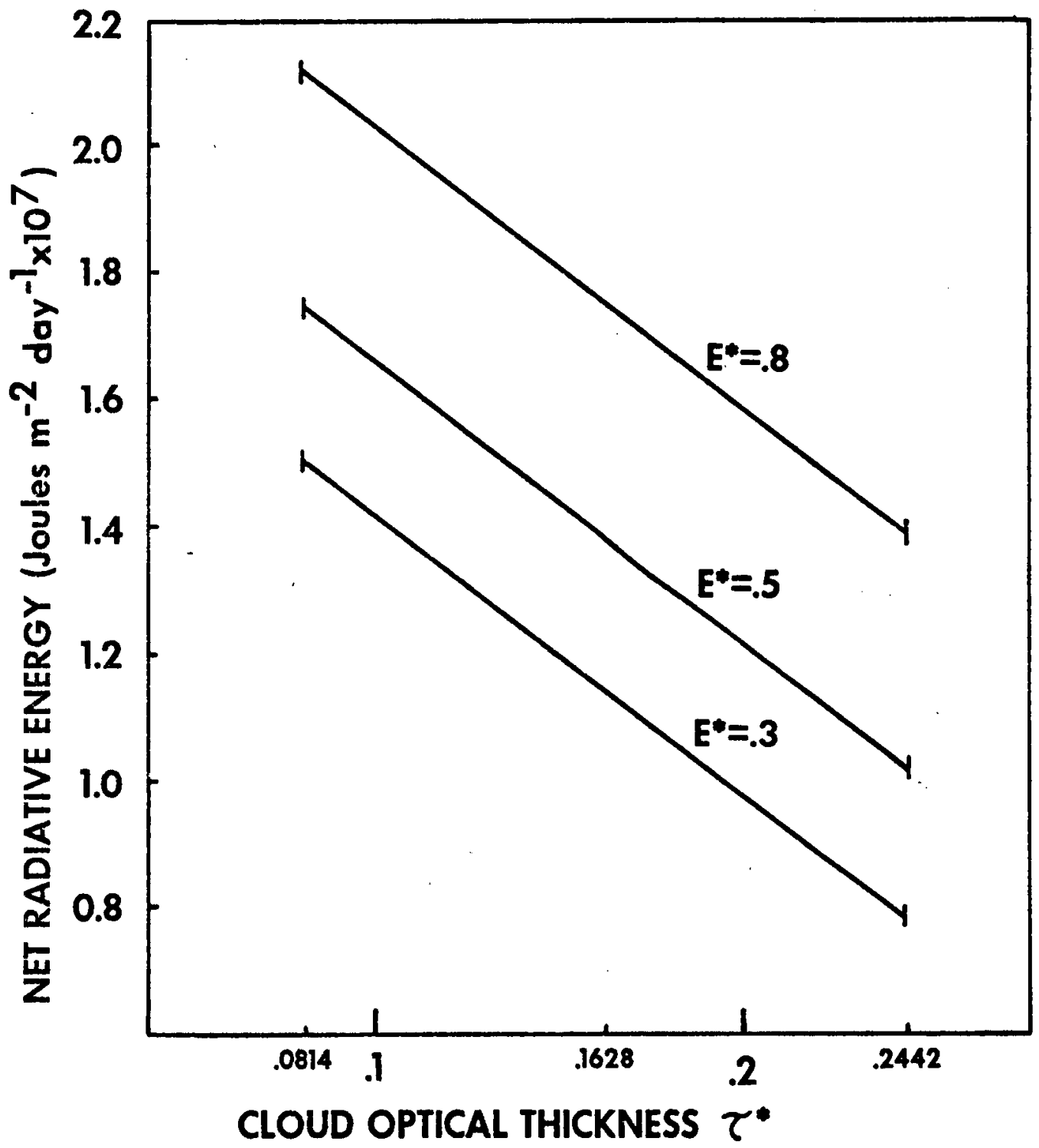

Figure 23. - Net radiative energy at the top of the atmosphere as a function of $\tau^{*}$ and $\varepsilon^{*}$ of the cirrus cloud. 


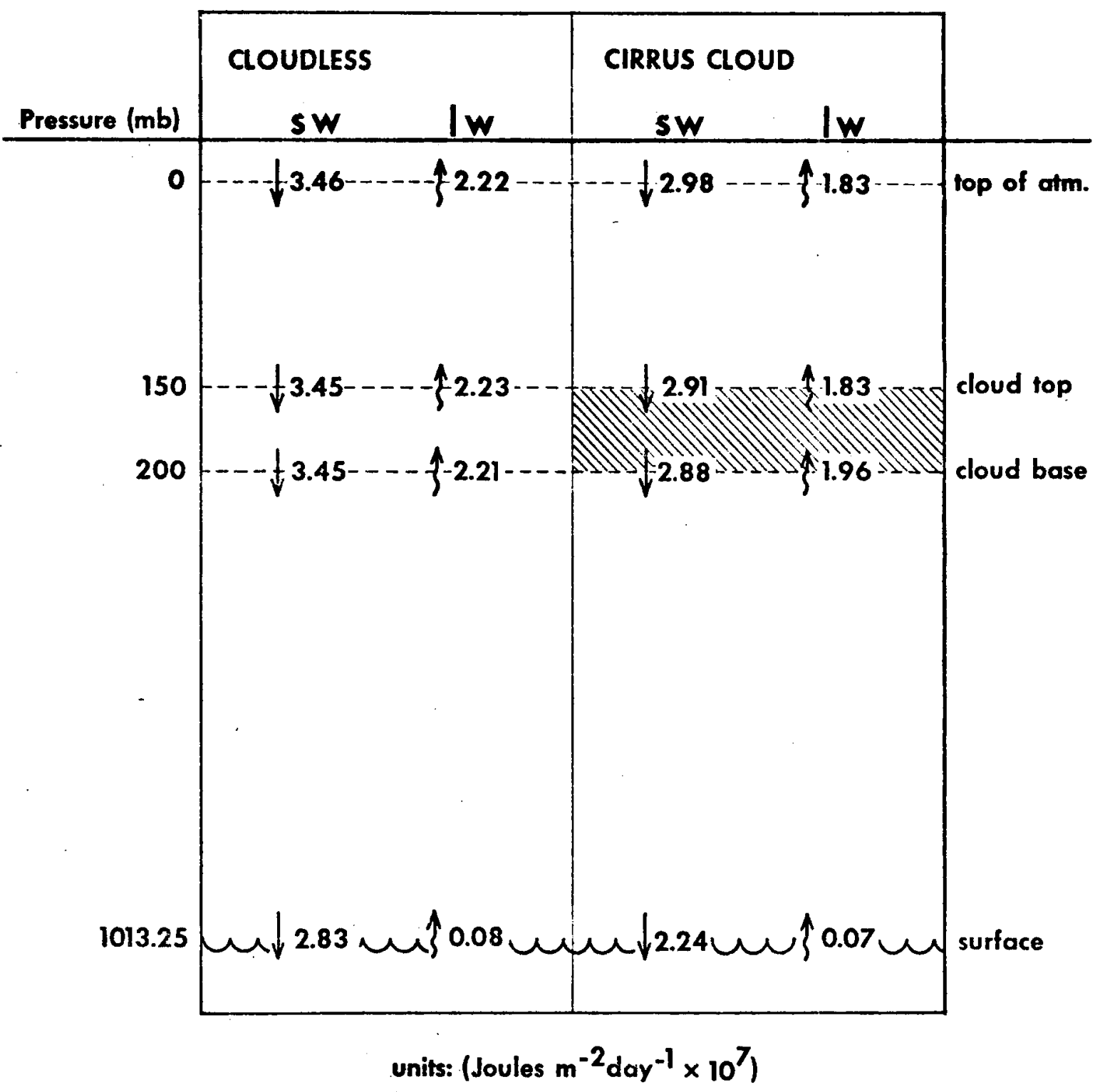

Figure 24a. - Shortwave and longwave daily radiative energy budgets for the cloudless atmosphere and with cirrus clouds present. 


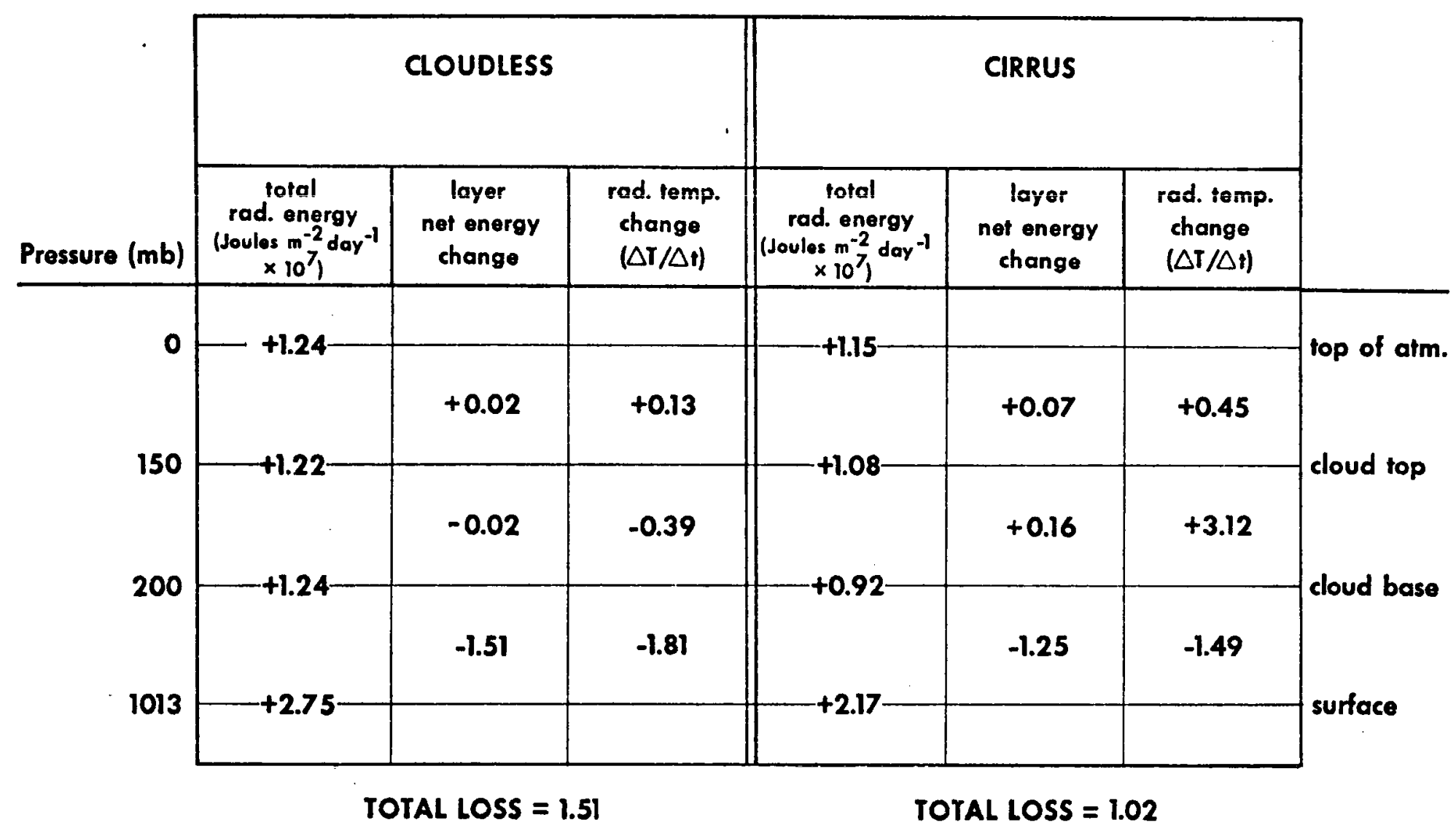

Figure 24b. - Total radiative energy, layer net energy change and radiative temperature change for cloudless, and cirrus conditions. 
suppressed relative to the divergence in the cloudless atmosphere. This is due to the increased downward longwave irradiance at the cirrus cloud base. The total loss through the atmosphere of $1.02 \times$ $10^{7}$ Joules $\mathrm{m}^{-2}$ day $^{-1}$ with this cirrus cloud present is $68 \%$ of the divergence in the cloudless atmosphere. Increasing the cirrus cloud's infrared emissivity to 0.8 decreases the total divergence to $27 \%$ of the cloudless sky value. A $2 \mathrm{~km}$ thick cloud located at $300 \mathrm{mb}$ with $\varepsilon^{*}=0.3$ reduces the total divergence to $74.0 \%$ of the cloudless value.

The divergence of total radiation in the atmosphere is given by the difference in the radiative energy at the top and at the bottom. Table 4 summarizes this energy divergence for three values of surface temperature and for probable radiative properties of $2 \mathrm{~km}$ thick cirrus clouds with a base pressure of $200 \mathrm{mb}$.

The importance of a cirrus cloud in the energetics of the atmosphere may be illustrated by comparing the release of energy to the atmosphere by precipitation processes in a cumulonimbus cloud with the suppression of radiative energy divergence caused by a cirrus cloud layer which may have been produced by the convective cell. Lopez (1972) has estimated the total rainfall expected from a typical cumulonimbus cloud to be $2.4 \mathrm{~cm}$ of water over a $2 \mathrm{~km}$ effective cloud radius. This is $3 \times 10^{11} \mathrm{gm}$ of liquid water with a total latent energy content of $7.50 \times 10^{14}$ Joules. The divergence of total radiation (from Table 4) with $\tau^{*}=0.1673$, $\varepsilon^{*}=0.5$ and $T_{S}=298 \mathrm{~K}$ is $0.94 \times 10^{7}$ Joules $\mathrm{m}^{-2}$ day $^{-1}$. This is a gain of $0.74 \times 10^{7}$ Joules $\mathrm{m}^{-2}$ day $^{-1}$ over the clear sky value. 
Table 4

DIVERGENCE OF TOTAL RADIATION IN THE TROPICAL ATMOSPHERE OVER AN OCEAN SURFACE WITH CIRRUS PRESENT

$$
\text { (Joules } \mathrm{m}^{-2} \text { day }^{-1} \times 10^{7} \text { ) }
$$

\begin{tabular}{|c|c|c|c|c|c|}
\hline$\tau^{\star}$ clear & $T_{s}$ & & & & \\
\hline & 294 & & & & \\
\hline & 298 & & & & \\
\hline & 302.6 & & & & \\
\hline$\tau^{*}=.0814$ & $T_{s}$ & $\varepsilon^{*}$ & .3 & .5 & .8 \\
\hline & 294 & & 1.23 & 0.99 & 0.62 \\
\hline & 298 & & 1.10 & 0.86 & 0.49 \\
\hline & 302.6 & & 0.94 & 0.70 & 0.33 \\
\hline$\tau^{*}=.1628$ & $T_{s}$ & $\underline{\varepsilon^{*}}$ & .3 & .5 & .8 \\
\hline & 294 & & 1.31 & 1.07 & 0.70 \\
\hline & 298 & & 1.18 & 0.94 & 0.57 \\
\hline & 302.6 & & 1.02 & 0.78 & 0.41 \\
\hline$\tau^{\star}=.2442$ & $T_{s}$ & $\underline{\varepsilon^{*}}$ & .3 & .5 & .8 \\
\hline & 294 & & 1.42 & 1.18 & 0.81 \\
\hline & 298 & & 1.29 & 1.05 & 0.68 \\
\hline & 302.6 & & 1.13 & 0.89 & 0.52 \\
\hline
\end{tabular}


From satellite observations by Vonder Haar (1969), the area of the cirrus canopy is estimated to be five times that of the cumulonimbus cloud. This cirrus cloud will generate radiatively in one day an energy excess over the clear sky value of $4.65 \times 10^{14}$ Joules or $62.0 \%$ of the latent energy content of the precipitation. Similar comparisons using the values of Table 5 give a minimum value of $31.8 \%$ for $\tau^{*}=.2442, \varepsilon^{*}=0.3, T_{S}=302.6 \mathrm{~K}$ and a maximum value of $99.7 \%$ for $\tau^{*}=0.0814, \varepsilon^{*}=0.8, T_{S}=294 \mathrm{~K}$. These maximum and minimum values may be considered to be equally probable since Davis (1970) shows no relationship between shortwave optical thickness and infrared emissivity for the cirrus clouds discussed above. 
V. CONCLUSION

The radiative effects of cirrus clouds in a tropical atmosphere have been quantitatively modelled in terms of two bulk radiative properties of the cloud: shortwave optical thickness and effective infrared emissivity. Single scattering of shortwave radiation is assumed because of the small values of optical thickness characteristic of cirrus clouds. The cloud is assumed to emit thermally as a grey body.

Examination of the vertical profiles of radiative temperature change in the atmosphere with cirrus clouds present indicates that cirrus clouds may be of major importance in determining the energy budget of the tropical atmosphere. This assertion is based upon the following results:

(1) The suppression, relative to the clear sky, of radiative energy divergence where a cirrus cloud is present causes in 24 hours an energy excess which is $31.8 \%$ to $99.7 \%$ of the latent heat release in a cumulonimbus cloud. This energy excess increases with increasing cloud infrared emissivity and with decreasing cloud temperature.

(2) For the tropical cases investigated in this study, the radiative cooling of every layer of the atmosphere, except the layer immediately above the cirrus cloud, is less than the corresponding radiative cooling in the cloudless atmosphere; in some instances by as much as $1^{0} \mathrm{C} \mathrm{day}^{-1}$.

(3) The presence of cirrus clouds decreases the total net radiative energy loss of the entire atmosphere to a value which is $78.5 \%$ to $21.9 \%$ of the loss from a cloudless atmosphere.

(4) The net radiative energy budget at the surface of the ocean shows a significant $21 \%$ decrease in the shortwave component when cirrus clouds 
are present while the net radiative energy at the top of the atmosphere is virtualiy unchanged by the presence of a typical cirrus cloud layer.

The radiative effects of any cirrus cloud may be derived using the methods presented in this study if the height and thickness of the cirrus cloud layer, the effective infrared emissivity, the ice particle concentration and the atmospheric temperature and moisture profiles are known. The radiative temperature changes of three layers encompassing the atmosphere are shown to be linearly dependent on the bulk radiative properties of the cirrus cloud. The parameterization of radiative divergence as a function of cloud thickness and base temperature has taken advantage of this linear variation.

Improvement of the model presented here depends on more and better measurements of the physical and radiative properties of cirrus clouds: radiometric measurements of $\varepsilon^{*_{\uparrow}}$ and $\varepsilon^{*} \downarrow$ will lend greater detail to the longwave results; determination of spectral emissivity will eliminate the problems associated with the grey body assumption; measurement of the cloud particle size distribution will allow better specification of the details of shortwave radiative transfer; shortwave transmissivity and albedo measurements will help to verify the model's theoretical predictions. 


\section{LITERATURE CITED}

Anderson, E. R., 1954: Energy Budget Studies. In Water-Loss Investigations: Lake Hefner Studies, Technical Report, U.S. Geological Survey, Professional Paper 269, Washington, pp. 71-119.

Bugno10, Dimitri S., 1960: On the Question of Multiple Scattering in the Troposphere, J. Geophys. Res., 65, pp. 879-884.

Cox, S. K., 1969: Observations of IR Effective Emissivities of Cloud Layers, Radiation in the Free Atmosphere, University of Wisconsin, Madison, pp. 27-37.

Cox, S. K., 1973: Infrared Heating Calculations with a Water Vapor Pressure Broadened Continuum, to be published in Q.J.R.M.S.

Danielson, R. E., D. R. Moore, and H. C. Van de Hulst, 1969: The Transfer of Visible Radiation Through Clouds. J. Atmos. Sci., 26, pp. 10781087.

Davis, P. A., 1970: Airborne Ruby-Tidar and Radiometric Measurements of Cirrus and Haze During BOMEX. Project 7334, Stanford Research Institute, California, $97 \mathrm{pp}$.

Davis, P. A., 1971: Applications of an Airborne Ruby-1idar During a BOMEX Program of Cirrus Observations. J. App1. Meteor., 10, pp. 1314-1323.

Drummond, A. J., and J. R. Hickey, 1971: Large Scale Reflection and Absorption of Solar Radiation by Clouds as Influencing Earth Radiation Budgets: New Aircraft Measurements, International Conference on Weather Modification, pp. 267-276.

Gergen, J. L., 1958: Blackness of Clouds as Determined from Radiation Measurements. Technical Report, Atmospheric Physics Program, University of Minnesota, 92 pages.

Goody, R. M., 1964: Atmospheric Radiation, I. Theoretical Basis, Oxford University Press, 436 pages.

Hansen, James E., 1969: Exact and Approximate Solutions for Multiple Scattering by Cloudy and Hazy Planetary Atmospheres. J. Atmos. Sci., 26, pp. 478-487.

Jacobowitz, H., 1970: Emission, Scattering and Absorption of Radiation in Cirrus Cloud Layers. Ph.D. Thesis, Massachusetts Institute of Technology, 181 pages.

Jacobowitz, H., 1971: A Method for Computing the Transfer of Solar Radiation Through Clouds of Hexagonal Ice Crystals. J. Quant. Spectrosc. Radiat. Transfer., 11, pp. 691-695. 


\section{LITERATURE CITED - Continued}

Kondratyev, K. Ya., 1969: Radiation in the Atmosphere. Academic Press, New York, 912 pages.

Kuhn, P. M., and H. K. Weickmann, 1969: High A7titude Radiometric Measurements of Cirrus. J. App1. Meteor., 8, pp. 147-154.

Liou, K., 1973: Transfer of Solar Irradiance Through Cirrus Cloud Layers., J. Geophys. Res., 78, pp. 1409-1418.

Lopez, Raul Erlando, 1972: Cumulus Convection and Large-Scale Circulations, Part I, C.S.U. Atmospheric Science Paper No. 188, Colorado State University, Fort Col1ins, 99 pages.

Manabe, S., and F. MolTer, 1961: On the Radiative Equilibrium and Heat Balance of the Atmosphere. Mon. Wea. Rev., 89, pp. 503-532.

Powe11, W. M., and Clarke, 1936: Reflection and Absorption of Daylight at the Surface of the Ocean, J. Optical Soc. America., 26, pp. 111-120.

Rieh1, Herbert, 1962: Radiation Measurements Over the Caribbean During the Autumn of 1960, J. Geophys. Res., 67, pp. 3935-3942.

U. S. Standard Atmosphere Supplements, 1966: For sale by the Superintendent of Documents, U. S. Government Printing Office, Washington, D. C. Price $\$ 4.00$.

Van de Hulst, H. C., 1957: Light Scattering by Sma11 Particles, John Wiley and Sons, New York, 469 pages.

Van de Hulst, H. C., and K. Grossman, 1968: Multiple Light Scattering in Planetary Atmospheres. The Atmospheres of Venus and Mars, Gordon and Breach Science Publishers, Inc., New York, 288 pages.

Vonder Haar, Thomas H., 1969: Meteorological Applications of Reflected Radiance Measurements from ATS I and ATS III, J. Geophys. Res., 74, pp. 5404-5412. 
APPENDIX A: Radiative Temperature Change of the Cloud Layer

The dependence of the radiative temperature change of a layer on the broad band emissivity of the layer is derived from the longwave boundary conditions presented in Figure 8 . The net irradiance at each level is formed by differencing the downward and upward fluxes:

(1A) $H_{n}(1)=H_{\uparrow}(1)-H_{\downarrow}(1)=\left(1-\varepsilon^{*}\right) H_{\uparrow}(2)+\varepsilon^{* B}(1)-H_{\downarrow}(1)$

(2A) $H_{n}(2)=H_{\uparrow}(2)-H_{\downarrow}(2)=H_{\uparrow}(2)-\left(1-\varepsilon^{*}\right) H_{\downarrow}(1)-\varepsilon^{*} B(2)$

where $B(1)$ is the black body radiation at the temperature of the cloud top. The subscript 2 refers to cloud base. Subtracting equation (1A) from equation (2A) gives after some rearranging

(3A) $H_{n}(2)-H_{n}(1)=\varepsilon^{*}\left\{\left[H_{\psi}(1)+H_{\uparrow}(2)\right]-[B(1)+B(2)]\right\}$

for a black cloud the difference in net flux is

(4A) $B_{n}(2)-B_{n}(1)=\{[H \uparrow(1)+H \uparrow(2)]-[B(1)+B(2)]\}$

The rate of radiative temperature change for the layer is proportional to the difference in the net fluxes at the top and bottom of the layer. Dividing (3A) by (4A) gives:

$$
(5 A) \quad \varepsilon^{*}=\frac{H_{n}(2)-H_{n}(1)}{B_{n}(2)-B_{n}(1)}=\frac{(\Delta T / \Delta t)}{(\Delta T / \Delta t)_{B L A C K}}
$$

rearranging:

(6A) $\frac{\Delta T}{\Delta t}=\varepsilon^{*}\left(\frac{\Delta T}{\Delta t}\right)$ 
APPENDIX B: Albedo of the Sea Surface for Global Radiation

Shortwave radiation from the sun reaches the surface of the earth partly as direct radiation and partly as diffuse radiation. The total albedo of a surface is given by the sum of the albedos for the direct and diffuse radiation weighted by the corresponding fractions of direct and diffuse energy.

(1B) $A_{\text {TOTAL }}=F_{\text {DIRECT }} * A_{\text {DIRECT }}+F_{\text {DIFFUSE }}$ * $A_{\text {DIFFUSE }}$ where $F$ is the fraction of direct and diffuse energy and $A$ is the corresponding albedo.

A plane water surface with a parallel beam unpolarized light incident has an albedo given by the Fresnel formula

$$
\text { (2B) } A(\theta)=\frac{1}{2} \frac{\sin ^{2}(\theta-R)}{\sin ^{2}(\theta+R)}+\frac{\tan ^{2}(\theta-R)}{\tan ^{2}(\theta+R)}
$$

where $\theta$ is the angle of incidence of the beam and $R$ is the angle of refraction.

$$
\frac{\sin \theta}{\sin R}=n=1.33 \text { for water with shortwave }
$$

radiation incident.

Equation (2B) gives a very good approximation to observations over a calm sea in clear weather [Kondratyev (1969) § 7.3].

The albedo of a water surface for diffuse radiation can be computed by integrating equation (2B) over all incident angles.

$$
\text { (3B) } A_{\text {DIFFUSE }}=2 \int_{0}^{\pi / 2} A(\theta) \cos \theta \cdot \sin \theta d \theta
$$

where $\theta$ is the solar zenith angle. The value of the integral is 0.066 . Measurements by Powe 11 and Clarke (1936) on an overcast day over the sea confirm this value. Table 1B presents the results of their measurements. 
TABLE IB.

\begin{tabular}{cccc}
\hline DATE & METEOROLOGICAL CONDITION & $\theta$ & $A$ \\
\hline 10 July 1935 & overcast, no wind & $70^{\circ}-53^{\circ}$ & 0.064 (average) \\
10 July 1935 & overcast, light wind & $16^{\circ}$ & 0.067 \\
10 July 1935 & overcast, light wind & $8^{\circ}-0^{\circ}$ & 0.065 (average) \\
\hline
\end{tabular}

The fractions of direct and diffuse energy reaching the surface with cirrus clouds present and under cloudless conditions, has been tabulated by Kondratyev (1969) as a function of solar zenith angle $\theta$. Table 2B presents the results of measurements.

TABLE 2B.

\begin{tabular}{|c|c|c|c|c|c|c|c|c|c|c|}
\hline Sol.ar Zenith Angle & 87.5 & 85 & 82.5 & 80 & 75 & 70 & 65 & 60 & 50 & 40 \\
\hline $\begin{array}{l}\text { Direct Energy } \\
\qquad\left[1 y \min ^{-1}\right]\end{array}$ & 0.00 & 0.00 & 0.00 & 0.00 & 0.04 & 0.11 & 0.21 & 0.32 & 0.60 & 0.90 \\
\hline $\begin{array}{l}\text { Diffuse Energy } \\
\qquad\left[1 y \min ^{-1}\right]\end{array}$ & 0.03 & 0.05 & 0.07 & 0.09 & 0.13 & 0.16 & 0.19 & 0.22 & 0.26 & 0.29 \\
\hline $\mathrm{F}_{\text {DIRECT }}$ & 0.00 & 0.00 & 0.00 & 0.00 & 0.24 & 0.41 & 0.53 & 0.59 & 0.70 & 0.76 \\
\hline$-\cdots-\cdots$ & --- & -- & CLOUD & LESS & -- & -- & --- & -- & -- & -- \\
\hline $\begin{array}{l}\text { Direct Energy } \\
{\left[\quad\left[1 y \min ^{-1}\right]\right.}\end{array}$ & 0.03 & 0.06 & 0.09 & 0.13 & 0.22 & 0.33 & 0.46 & 0.59 & 0.84 & 1.10 \\
\hline $\begin{array}{l}\text { Diffuse Energy } \\
{\left[\quad\left[1 \mathrm{y} \min ^{-1}\right]\right.}\end{array}$ & 0.02 & 0.03 & 0.04 & 0.05 & 0.07 & 0.08 & 0.09 & 0.10 & 0.11 & 0.12 \\
\hline $\mathrm{F}_{\mathrm{DIRECT}}$ & 0.60 & 0.67 & 0.69 & 0.72 & 0.76 & 0.80 & 0.84 & 0.86 & 0.88 & 0.90 \\
\hline
\end{tabular}


Equation (1B) was then evaluated at each zenith angle $\theta$ to give the albedo of the sea surface for global radiation which is shown in Figure (1B). The solid line is the cloudless case and the broken line is the albedo when cirrus clouds are present. 


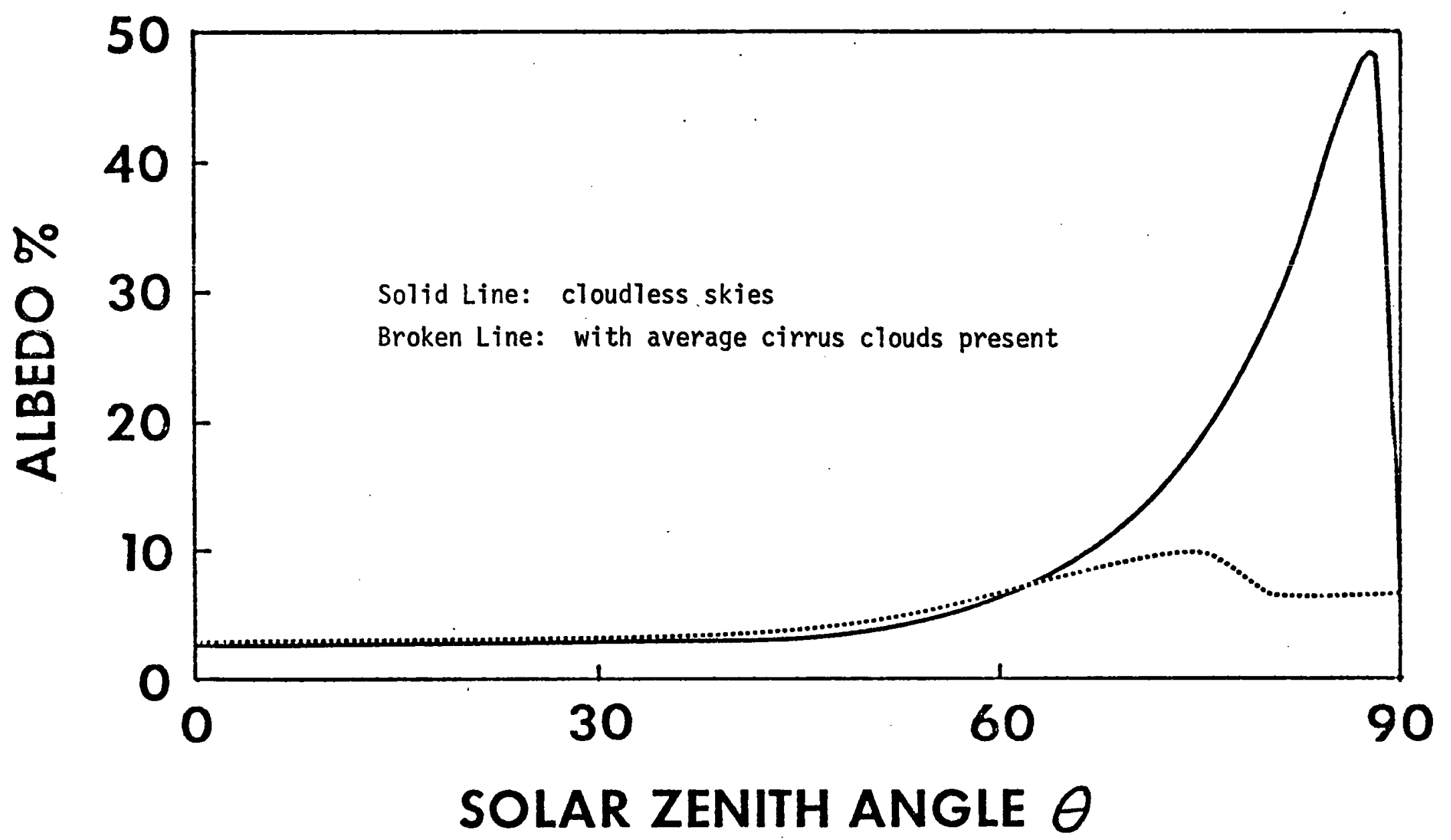

Figure 1B. - Albedo of the sea surface for global radiation vs. solar zenith angle. 


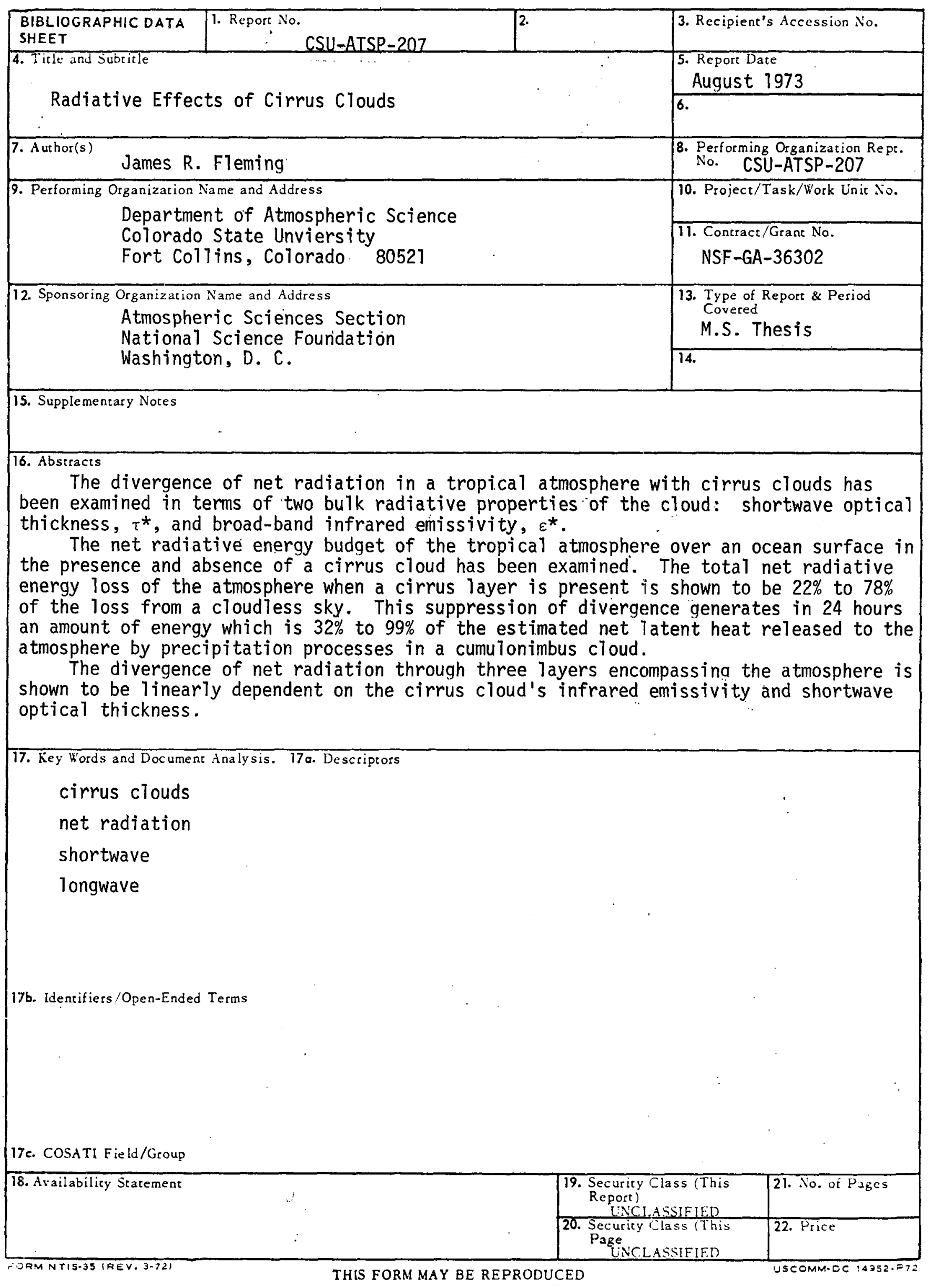

